ARTICLE

Received 16 Jul 2014 | Accepted 12 Dec 2014 | Published 30 Jan 2015

DOI: $10.1038 /$ ncomms7137

OPEN

\title{
Augmented AMPK activity inhibits cell migration by phosphorylating the novel substrate Pdlim5
}

Yi Yan ${ }^{1, \star}$, Osamu Tsukamoto ${ }^{1, \star}$, Atsushi Nakano ${ }^{2}$, Hisakazu Kato ${ }^{1}$, Hidetaka Kioka ${ }^{3}$, Noriaki Ito ${ }^{3}$, Shuichiro Higo ${ }^{3}$, Satoru Yamazaki ${ }^{4}$, Yasunori Shintani ${ }^{1}$, Ken Matsuoka ${ }^{3}$, Yulin Liao ${ }^{5}$, Hiroshi Asanuma ${ }^{2}$, Masanori Asakura ${ }^{2}$, Kazuaki Takafuji6, Tetsuo Minamino ${ }^{3}$, Yoshihiro Asano ${ }^{3}$, Masafumi Kitakaze ${ }^{2}$ \& Seiji Takashima1,7

\begin{abstract}
Augmented AMP-activated protein kinase (AMPK) activity inhibits cell migration, possibly contributing to the clinical benefits of chemical AMPK activators in preventing atherosclerosis, vascular remodelling and cancer metastasis. However, the underlying mechanisms remain largely unknown. Here we identify PDZ and LIM domain 5 (Pdlim5) as a novel AMPK substrate and show that it plays a critical role in the inhibition of cell migration. AMPK directly phosphorylates Pdlim5 at Ser177. Exogenous expression of phosphomimetic S177D-Pdlim5 inhibits cell migration and attenuates lamellipodia formation. Consistent with this observation, S177D-Pdlim5 suppresses Rac1 activity at the cell periphery and displaces the Arp2/3 complex from the leading edge. Notably, S177D-Pdlim5, but not WT-Pdlim5, attenuates the association with Rac1-specific guanine nucleotide exchange factors at the cell periphery. Taken together, our findings indicate that phosphorylation of Pdlim5 on Ser177 by AMPK mediates inhibition of cell migration by suppressing the Rac1-Arp2/3 signalling pathway.
\end{abstract}

\footnotetext{
${ }^{1}$ Department of Medical Biochemistry, Osaka University Graduate School of Medicine, 2-2 Yamadaoka, Suita, Osaka 565-0871, Japan. ${ }^{2}$ Depertment of Clinical Research and Development, National Cerebral and Cardiovascular Center Research Institute, Suita, Osaka 565-8565, Japan. ${ }^{3}$ Department of Cardiovascular Medicine, Osaka University Graduate School of Medicine, 2-2 Yamadaoka, Suita, Osaka 565-0871, Japan. ${ }^{4}$ Department of Cell Biology, National Cerebral and Cardiovascular Center Research Institute, Suita, Osaka 565-8565, Japan. ${ }^{5}$ Department of Cardiology, Nanfang Hospital, Southern Medical University, 1838 North Guangzhou Avenue, 510515 Guangzhou, China. ${ }^{6}$ Center for Research Education, Osaka University Graduate School of Medicine, 2-2 Yamadaoka, Suita, Osaka 565-0871, Japan. ${ }^{7}$ Japan Science and Technology Agency-Core Research for Evolutional Science and Technology (CREST), Kawaguchi 332-0012, Japan. * These authors contributed equally to this work.. Correspondence and requests for materials should be addressed to O.T. (email: tsuka@medbio.med.osaka-u.ac.jp).
} 
MP-activated protein kinase (AMPK), generally considered an energy sensor kinase, requires AMP for activation $^{1}$. Recently, a growing body of evidence has revealed that AMPK also plays a key role in the establishment of cell polarity and motility ${ }^{2,3}$. We previously reported that AMPK regulates cell migration by controlling microtubule dynamics through phosphorylation of a cytoplasmic linker protein-170 $\left(\right.$ CLIP-170) ${ }^{4}$. Moreover, recent studies have implicated AMPK in the regulation of actin cytoskeleton dynamics and reorganization at the plasma membrane ${ }^{5,6}$. Thus, AMPK is predicted to regulate cell migration by controlling both microtubule and actin-filament dynamics.

Cell migration is a physically integrated molecular process that begins with dynamic polarization and formation of lamellipodia, membrane protrusions at the leading edges of cells ${ }^{7}$. Rac1, a Rhofamily small GTPase, is a key upstream regulator of actin dynamics and organization, and is necessary for the formation of persistent lamellipodia leading to directional cell migration ${ }^{8,9}$. Once Racl is activated by guanine nucleotide exchange factors (GEFs) at the leading edge, the activated form (GTP-bound Rac1) recruits a complex containing its downstream effector WiskottAldrich Syndrome protein family verprolin homologous to the plasma membrane, leading in turn to activation of the actinrelated protein 2/3 (Arp2/3) complex ${ }^{10,11}$. Activated Arp2/3 complex functions as an efficient nucleator ${ }^{10,11}$ to organize the branched actin-filament network involved in formation of lamellipodia, a critical step in cell migration.

Some drugs in clinical use have the potential to indirectly activate AMPK. These compounds have been convincingly shown to prevent atherosclerosis, vascular remodelling, and tumour invasion and metastasis ${ }^{12-17}$, processes in which dysregulated cell migration contributes to the development and progression of diseases. Accordingly, the clinically beneficial effects of chemical AMPK activators can be partially attributed to inhibition of cell migration via augmentation of AMPK activity. However, the mechanisms by which augmented AMPK activity inhibits cell migration remain largely unknown.

In this study, we identified PDZ and LIM domain 5 (Pdlim5) ${ }^{18}$ as a novel substrate of AMPK; Pdlim5 is directly phosphorylated by AMPK at Ser177. This phosphorylation results in displacement of Rho GEF 6 (Arhgef6), a Rac1/Cdc42-specific GEF and also known as p21-activated kinase-interacting exchange factor- $\alpha$ PIX, from the leading edge of the cell by disrupting the association between Pdlim5 and Arhgef6. Displacement of Arhgef6 suppresses Racl activity and the disappearance of Arp $2 / 3$ complex from the cell periphery, leading to defects in lamellipodia formation and inhibition of directional cell migration. We propose that Pdlim5 is the main signalling molecule that regulates cell migration in the context of augmented AMPK activity.

\section{Results}

Pdlim5 is phosphorylated at Ser177 by AMPK. Our group has worked on AMPK for many years. To estimate the AMPK activity level in vivo, we often monitor phospho-acetyl-CoA carboxylase (pACC), a well-known substrate of AMPK whose level generally reflects the AMPK activity ${ }^{4}$. When we treated $\mathrm{C} 2 \mathrm{C} 12$ cells with AMPK activators such as 5-aminoimidazole-4-carboxyamide ribonucleoside (AICAR), A-769662 and 2-deoxy-D-glucose (2DG), we serendipitously observed reproducible and specific induction of a protein with an apparent molecular mass of $64 \mathrm{kDa}$ (p64) that cross-reacted with a commercially available antibody against pACC (Fig. 1a and Supplementary Fig. 1a,b, respectively). Three-step column chromatography followed by mass-spectrometric analysis revealed that p64 was likely to be
Pdlim5 (ref. 19; Fig. 1b,c). To confirm this, we generated three polyclonal antibodies against mouse Pdlim5 (Supplementary Fig. 1c) and designed two different small interfering RNAs (siRNAs) against the Pdlim5 messenger RNA (Supplementary Fig. 1d). When we treated $\mathrm{C} 2 \mathrm{C} 12$ cells with siPdlim5, the p64 band disappeared (Fig. 1d). Therefore, we concluded that p64 is indeed Pdlim5. Furthermore, as the p64 band, probably representing a phosphorylated form of Pdlim5, was detected exclusively after AMPK activation, we speculated that Pdlim5 is a substrate of AMPK. Pdlim5, also known as Enigma homolog protein, is an $\alpha$-actinin-binding protein that possesses a PDZ domain at its amino terminus and three LIM motifs at its carboxy terminus ${ }^{19}$. Pdlim5 anchors to the actin cytoskeleton via its PDZ domain and recruits LIM-associated proteins to actin filaments ${ }^{20}$. To narrow down the location of the phosphorylation site, we transfected wild-type (WT) Pdlim5 or deletion mutants (Supplementary Fig. 2a) into HEK293T cells, and then treated the transfectants with AICAR. A mutant truncated after N184 cross-reacted with the pACC antibody following AMPK activation (Supplementary Fig. 2b), whereas a mutant truncated after N160 did not, indicating that the phosphorylation site resides within the N-terminal segment of Pdlim5 between Ala161 and Asn 184 .

Next, we introduced Ser-to-Ala or Thr-to-Ala point mutations into this putative phosphorylation segment (Fig. 2a). Both the S175A and S177A mutants lost cross-reactivity with pACC antibody (Fig. 2a). Next, we performed in vitro phosphorylation assays on recombinant FLAG-tagged Pdlim5 (WT, S175A and S177A). The results revealed that incorporation of $\left[\gamma-{ }^{32} \mathrm{P}\right] \mathrm{ATP}$ into Pdlim5 was inhibited only in the S177A mutant, indicating that Ser177 is the unique phosphorylation site of Pdlim5 (Fig. 2b). We confirmed direct phosphorylation of Pdlim5 at Ser177 by AMPK by in vitro phosphorylation assay using recombinant glutathione S-transferase (GST)-fused Pdlim5 (WT or S177A) expressed in Escherichia coli (Supplementary Fig. 2c,d), and subsequently by using an antibody we generated (Supplementary Fig. 2e) against Ser177-phosphorylated Pdlim5 (Ab-pS177) (Fig. 2c). The amino-acid sequence surrounding Ser177 matches the consensus sequence for AMPK phosphorylation sites (Supplementary Fig. 2f) and also exhibits a high similarity with the phosphorylation site of ACC (Supplementary Fig. 2f). Furthermore, Ser177, but not Ser175, is highly conserved only in mammals (Supplementary Fig. 3). Next, we confirmed that endogenous Pdlim5 is phosphorylated at Ser177 on AMPK activation in vivo in vascular smooth muscle cells (vSMCs) (Fig. 2d and Supplementary Fig. 4). Finally, we investigated whether Pdlim5 is phosphorylated in response to an acute physical stimulus. Hypoxia $\left(1 \% \mathrm{O}_{2}\right.$ for $\left.2 \mathrm{~h}\right)$ activated AMPK and induced Ser177-phosphorylation of Pdlim5 (Fig. 2e). Together, these data indicated that augmented AMPK activity induces Ser177 phosphorylation of Pdlim5 both in vitro and in vivo.

Ser177 phosphorylation of Pdlim5 inhibits cell migration. To accurately assess the functions of Ser177 phosphorylation of Pdlim5 by AMPK, it is essential to use a system that eliminates any effects associated with AMPK activation other than Ser177 phosphorylation. For this purpose, we established the knockdown-rescue (KDR) system in vSMCs by depleting endogenous Pdlim5 and simultaneously expressing siPDLIMresistant enhanced green fluorescent protein (EGFP)-tagged Pdlim5 (Fig. 3a). For this purpose, we treated cells with siRNA oligonucleotides targeting the $3^{\prime}$-untranslated region mRNA of mouse Pdlim5 (siPdlim5-2) and infected them with adenovirus encoding EGFP-WT-, EGFP-S177A- or EGFP-S177D-Pdlim5, yielding cells we designated KDR/WT-, KDR/S177A- or KDR/ 
a

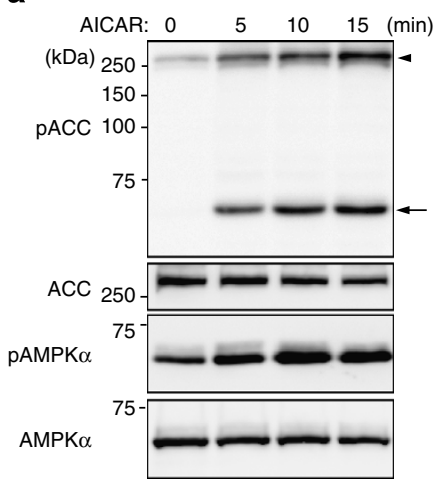

d

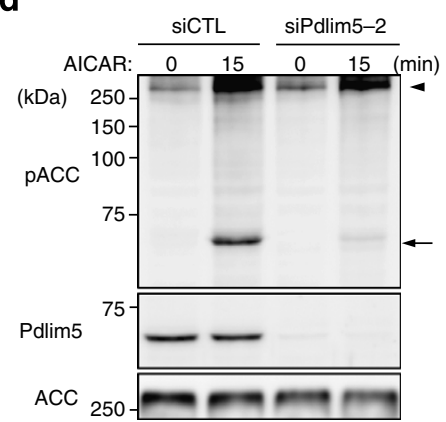

b

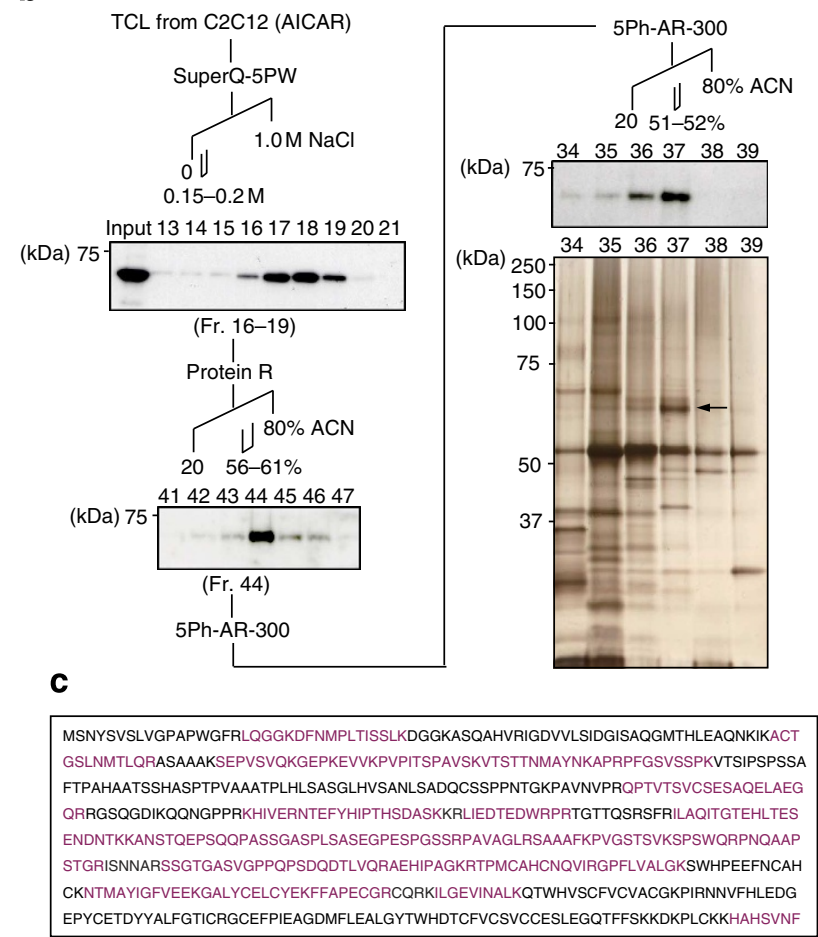

Figure 1 | Pdlim5 is a substrate of AMPK. (a) C2C12 cells were stimulated with AICAR ( $2 \mathrm{mM}) 12 \mathrm{~h}$ after serum starvation. Total cell lysates (TCLs) were harvested 0, 5, 10 and $15 \mathrm{~min}$ after stimulation and subjected to immunoblotting with anti-pACC antibody. In addition to the predicted band for pACC (arrowhead), a cross-reacting band, p64 (arrow), was detected in a time-dependent manner after AICAR treatment. (b) A schematic representation of the purification and identification of p64. TCLs from C2C12 cells treated with AICAR were subjected to three-step column chromatography (superQ-5PW, protein R and 5Ph-AR-300). p64 was detected using the anti-pACC antibody in each step. Images were obtained by immunoblotting and silver staining. The identified band (arrow in silver-stained gel), fractionated by reverse-phase HPLC (5Ph-AR-300), was excised and analysed by mass spectrometry. (c) Amino acid sequence of Pdlim5. Sequence analysis by MALDI-Qq-TOF MS/MS revealed the target protein to be Pdlim5. Matching amino acids are shown in magenta letters. (d) C2C12 cells treated with siRNA (siCTL or siPdlim5-2) were stimulated with AICAR and TCLs were subjected to immunoblotting with pACC and Pdlim5 antibodies (Ab229-2). Arrowhead and arrow denote pACC and p64, respectively.

S177D-Pdlim5, respectively. This KDR system successfully replaced endogenous Pdlim5 with EGFP-Pdlim5 at physiological expression levels in vSMCs (Fig. 3a). Exogenously expressed EGFP-Pdlim 5 co-localized with $\alpha$-actinin and F-actin structures (Fig. 3b), a pattern similar to that of endogenous Pdlim5 (Supplementary Fig. 5), although EGFP-Pdlim5 appeared in the cytoplasm at much higher levels than the endogenous protein. Importantly, EGFP-WT-Pdlim5 was phosphorylated by AMPK in the same manner as endogenous Pdlim5, whereas EGFPS177A-Pdlim5, an unphosphorylatable mutant, was not phosphorylated (Fig. 3c). EGFP-S177D-Pdlim5, a phosphomimetic mutant, was recognized by the Ab-pS177 antibody even in the absence of AICAR stimulation (Fig. 3c). This result indicates that phosphorylated WT-Pdlim5 and the S177D-Pdlim5 mutant are structurally similar. Thus, even in the absence of AMPK activators, exogenously expressed EGFP-S177D-Pdlim5 behaved similar to endogenous Pdlim5 phosphorylated by AMPK. It is particularly noteworthy that the KDR system can exclude any effects associated with AMPK activation other than Ser177 phosphorylation of Pdlim5.

AMPK activation inhibits cell migration; to investigate whether this effect is mediated by Pdlim5 phosphorylation at Ser177, we performed a scratch assay using the $\mathrm{KDR} / \mathrm{VSMC}$ system in the absence of AMPK activators. KDR/S177D-Pdlim5 cells exhibited a marked delay in scratch closure relative to both KDR/WT- and KDR/S177A-Pdlim5 cells (Fig. 3d,e and Supplementary Movie 1). By tracking the movement of individual cells, we could calculate the path length (L) and displacement (D) of individual cells
(Fig. 3f). KDR/S177D-Pdlim5 cells exhibited a lower migration speed (defined as L/total trajectory time) and less directionality (defined as D/L) than KDR/WT- and KDR/S177A-Pdlim5 cells (Fig. 3g,h). Next, we performed a scratch assay in the presence of AMPK activator (Supplementary Fig. 6). Compared with KDR/ WT- and KDR/S177D-Pdlim5 cells, the degree of cell migratory inhibition was lower in KDR/S177A-Pdlim5 cells (Supplementary Fig. 6). Furthermore, we examined single-cell migration in the absence of AMPK activators. Consistent with the results of the scratch assay, KDR/S177D-Pdlim5 cells exhibited impaired cell migration (Supplementary Fig. 7a,b and Supplementary Movie 2).

Next, we examined the AMPK specificity of the phenotypes observed in KDR cells using AMPK $\alpha 1^{-1-} \alpha 2^{-7-}$ mouse embryonic fibroblasts (AMPK $\alpha$-null MEFs). First, we confirmed that Pdlim5 phosphorylation by AMPK activators was completely blocked in AMPK $\alpha$-null MEFs (Fig. 4a and Supplementary Fig. 8). Moreover, a scratch assay confirmed that AMPK activator-induced cell migratory inhibition was abolished in AMPK $\alpha$-null MEFs (Fig. 4b-d and Supplementary Movie 3). For further confirmation, we constructed a Pdlim5 knockout vSMC (Pdlim5 ${ }^{-1-}$ vSMC) line by genome editing, using the CRISPR/ Cas9 system (Supplementary Fig. 9), and established a knockout and rescue cell system by expressing EGFP-Pdlim5 (WT, S177A or S177D) at physiological level by adenovirus transduction of knockout cells (Supplementary Fig. 10a). When the knockout and rescue cells were subjected to a cell migration assay (Supplementary Fig. 10b-d), the results were consistent with 
a

(aa) 161184
\begin{tabular}{|l|l|l|l|l|}
\hline PDZ & 161 & & \\
\hline
\end{tabular}

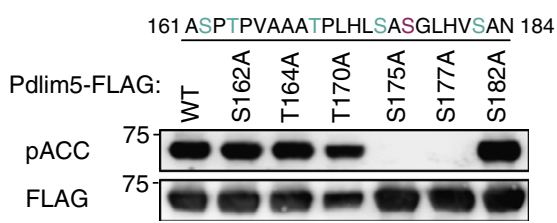

C

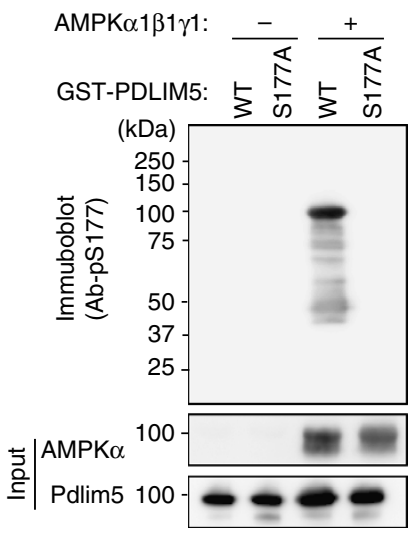

b

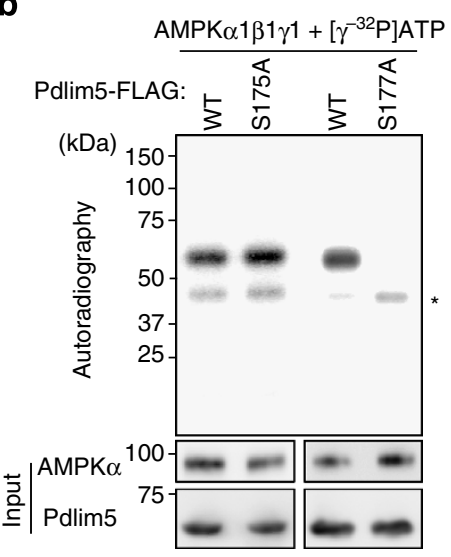

e d

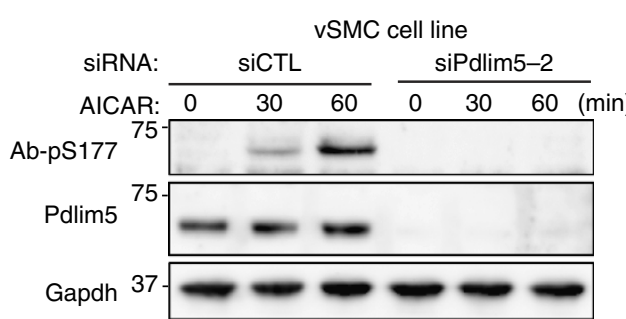

Hypoxia

$\left(1 \% \mathrm{O}_{2}, 2 \mathrm{~h}\right)$

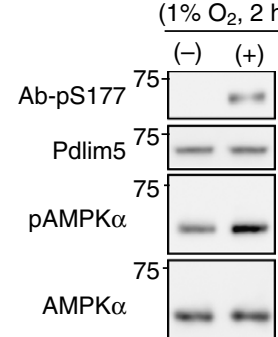

Figure 2 | Pdlim5 is directly phosphorylated at Ser177 by AMPK. (a) Diagrams of point-mutated Pdlim5. HEK293T cells were transiently expressed with each point-mutant of cFLAG-tagged Pdlim5 and treated with AICAR ( $2 \mathrm{mM}$ ) for $15 \mathrm{~min}$. Proteins purified on anti-FLAG M2 agarose were subjected to immunoblotting with anti-pACC or anti-FLAG antibody. (b) In vitro assay for AMPK phosphorylation. Recombinant cFLAG-tagged Pdlim5 (WT, S175A or S177A) from HEK293T cells was incubated with baculovirus-expressed recombinant AMPK in the presence of [ $\gamma$ - ${ }^{32}$ P]ATP and then subjected to autoradiography. Asterisk denotes bands of $\alpha$-subunit of recombinant AMPK. (c) In vitro phosphorylation assay by AMPK. Recombinant GST-tagged Pdlim5 proteins (WT and S177A) were incubated with or without recombinant AMPK and then subjected to immunoblotting with Ab-pS177. (d) vSMCs transfected either with siCTL or siPdlim5-2 were treated with AICAR ( $2 \mathrm{mM})$ for the indicated times, and TCLs were subjected to immunoblotting with Ab-pS177. (e) vSMCs were exposed to physiological hypoxia $\left(1 \% \mathrm{O}_{2}\right.$ for $\left.2 \mathrm{~h}\right)$. TCLs were subjected to immunoblotting with the indicated antibodies.

those obtained with the KDR cells. Together, these results indicated that Ser177 phosphorylation of Pdlim5 by AMPK inhibits cell migration.

Ser177 phosphorylation altered actin architectures. To elucidate the mechanism by which Ser177 phosphorylation of Pdlim5 inhibits cell migration, we monitored morphological changes in KDR cells. In all three types of KDR cells, EGFP-Pdlim5 protein co-localized with F-actin at the cell periphery and on stress fibres (Fig. 5a), indicating that co-localization of Pdlim5 with actin was not influenced by the phosphorylation state of Ser177. However, KDR/WT- and KDR/S177A-Pdlim5 cells displayed smooth lamellipodia-like edges, whereas KDR/S177D-Pdlim5 cells exhibited attenuated lamellipodia formation and jagged edges with excessive filopodia-like protrusions and ventral stress fibres (Fig. 5a and Supplementary Fig. 11a). In addition, both KDR/ WT- and KDR/S177A-Pdlim5 cells contained small and scattered spots of focal adhesions at the junction between the lamellipodia and lamella; by contrast, in KDR/S177D-Pdlim5 cells, focal adhesions were displaced to the edge of the cell and significantly enlarged in size (Fig. 5a and Supplementary Fig. 11b). To determine whether the morphological changes observed in KDR/ S177D-Pdlim5 cells were related to Ser177 phosphorylation of Pdlim5, we performed time-lapse imaging before and after treatment with AMPK activator (Fig. 5b,c). In KDR/WT-Pdlim5 cells, but not in KDR/S177A-Pdlim5 cells, AMPK activation induced defective lamellipodia formation and promoted expansion of the EGFP signals from the side opposite the lamellae towards the cell centre (Fig. 5b,c and Supplementary Movie 4), a pattern resembling the growth of dorsal stress fibres. This morphological change was similar to that observed in KDR/S177DPdlim5 cells. Furthermore, AMPK activation induced defective lamellipodia and enhanced stress fibre formation in WT-MEFs, but not AMPK $\alpha$-null MEFs (Fig. 4e). These findings suggested that Ser177 phosphorylation of Pdlim5 by augmented AMPK activity induced attenuation of lamellipodia and promoted formation of stress fibres from the cell periphery. Furthermore, these observations suggested that morphological changes of the actin architecture were initiated near the cell periphery.

Ser177 phosphorylation altered Arp2/3 complex localization. Accordingly, we focused on the Arp2/3 complex, because it is one of the major actin nucleators at the cell periphery and plays a key role in lamellipodia formation ${ }^{10}$. Expression levels of Arp $2 / 3$ complex were comparable among the various types of KDR cells (Supplementary Fig. 12a). However, the intracellular localization of Arp $2 / 3$ complex was markedly altered in both spreading and polarized cells (Fig. 6). In spreading cells, KDR/WT-Pdlim5 cells exhibited highly uniform lamellipodia throughout the cell edge, where Arp3 predominantly localized (Fig. 6a). By contrast, KDR/ S177D-Pdlim5 cells exhibited filopodia-like protrusions instead of lamellipodia, and localization of Arp3 shifted from the cell edge to a cytoplasmic distribution (Fig. 6a). A similar pattern was observed in polarized cell: Arp3 localized in actin-rich 
a

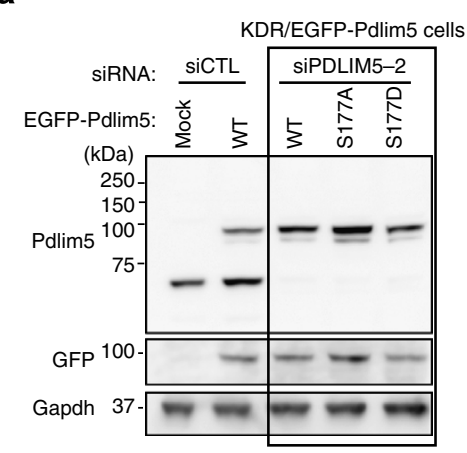

C

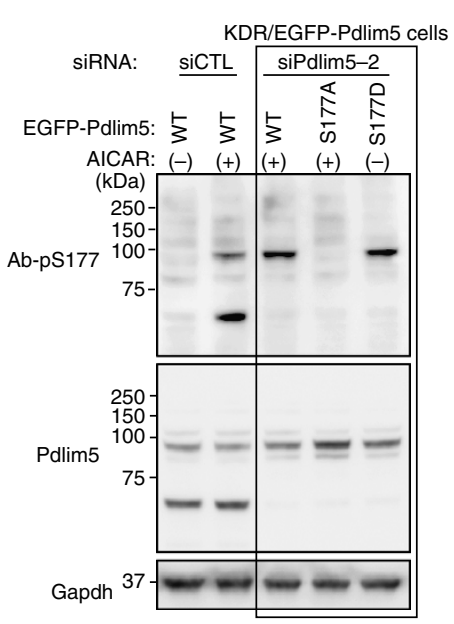

e

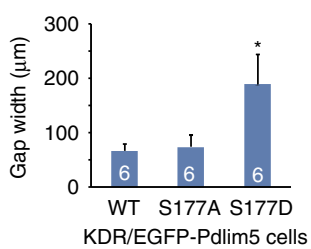

b

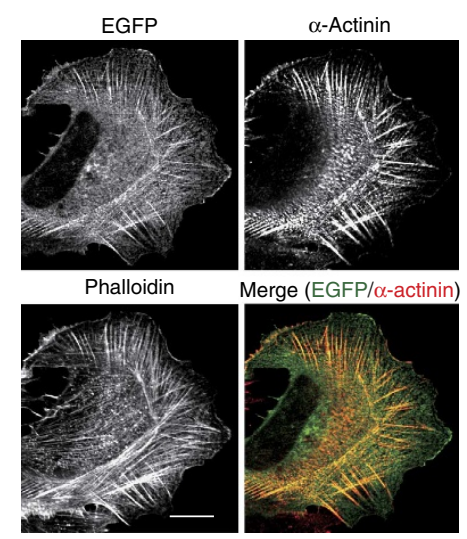

d
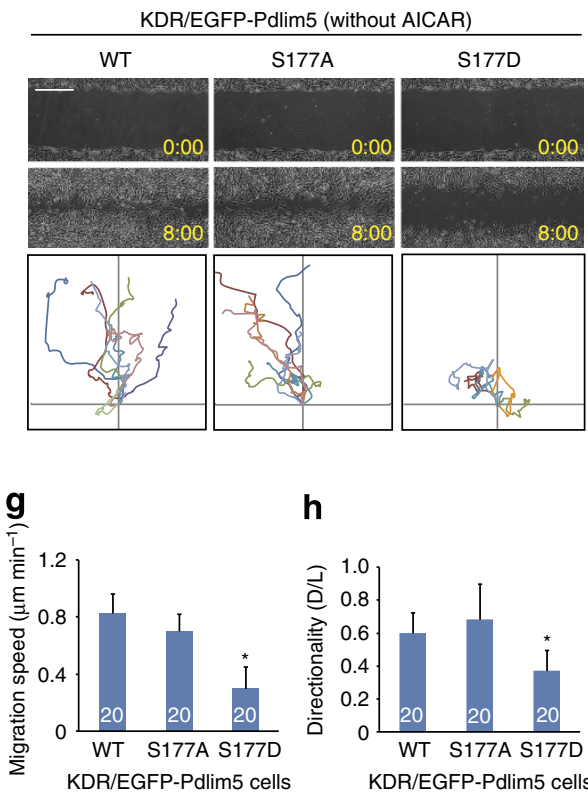

h

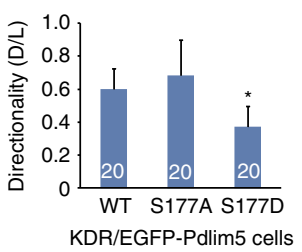

Figure 3 | Ser177 phosphorylation of Pdlim5 by AMPK inhibited directional migration of vSMCs. (a) Establishment of the KDR system for Pdlim5 in vSMCs. vSMCs were transfected with either siCTL or siPdlim5-2. siPdlim5-2-resistant EGFP-Pdlim5 (WT, S177A or S177D) was added back by adenoviral-mediated gene delivery. TCLs were subjected to immunoblotting. (b) GFP and immunostained images of KDR/EGFP-WT-Pdlim5 cells stained with a $\alpha$-actinin antibody and phalloidin. Scale bar, $10 \mu \mathrm{m}$. (c) KDR/EGFP-Pdlim5 (WT and S177A) cells were treated with AICAR for 60 min, whereas KDR/EGFP-S177D-Pdlim5 cells were not treated with AICAR. TCLs from each cell were subjected to immunoblotting. (d) Scratch assay of KDR/EGFPPdlim5 cells. Phase-contrast microscopy images of KDR/Pdlim5 cells (WT, S177A and S177D) before and $8 \mathrm{~h}$ after scratching in the absence of AICAR. The bottom row of each panel shows analysis of migration paths over $8 \mathrm{~h}$. The origins of migration of each cell were superimposed at [0, 0]. Scale bar, $0.5 \mathrm{~mm}$. (e) Bar graph showing the gap width $8 \mathrm{~h}$ after scratching (from d). (f) Demonstration of path length (L) and displacement (D) for calculation of migration velocity and directionality. (g) Bar graph showing migration speed of each cell (from $\mathbf{d}$ ). (h) Bar graph showing migration directionality of each cell (from d). Numbers in the bars indicate $n$. Data are representative of means \pm s.e.m. from three independent experiments. Significance of differences between series of results was assessed using one-way analysis of variance, followed by a post-hoc comparison with Dunnett's method for multiple comparisons. ${ }^{\star} P<0.01$ compared with WT.

lamellipodia in KDR/WT-Pdlim5 cells, but was distributed diffusely throughout the cytoplasm in KDR/S177D-Pdlim5 cells (Fig. 6b). These findings indicated that Ser177 phosphorylation of Pdlim5 impairs the function of the Arp $2 / 3$ complex by altering the localization of the complex from the cell edge to the cytoplasm. Consistent with this, vSMCs defective for Arp2/3 complex due to knockdown of the Arpc2 subunit exhibited a phenotype very similar to that of DR/S177D-Pdlim5 cells (Supplementary Fig. 12b,c). On the other hand, mammalian diaphanous (mDia), another actin nucleator, persisted at the leading edge of the cells (Supplementary Fig. 13).
Ser177 phosphorylation of Pdlim5 decreased Rac1 activity. Next, we measured the activities of Rac1, an upstream regulator of the Arp $2 / 3$ complex that is required for lamellipodia formation $^{8,9}$. GTP-bound active Rac1 was significantly reduced in KDR/S177D-Pdlim5 cells relative to KDR/WT- and KDR/S177APdlim5 cells (Fig. 7a). Furthermore, we carried out imaging of Racl activity in living cells, using vSMCs stably expressing the Raichu-Rac1 probe (vSMC- $R_{\text {Rac1 }}$ cells), which is based on the principle of fluorescence resonance energy transfer (FRET) biosensors $^{21}$. We established the KDR system in vSMC-R $\mathrm{R}_{\mathrm{Rac}}$ cells, as described above, except that siPDLIM-resistant Pdlim5 
a

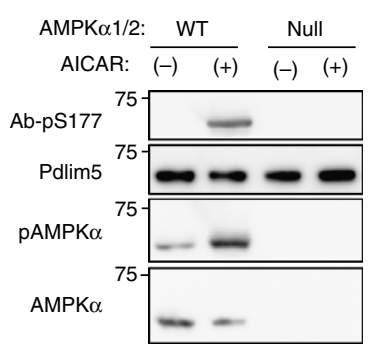

C

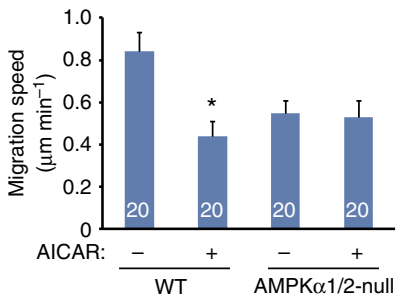

d

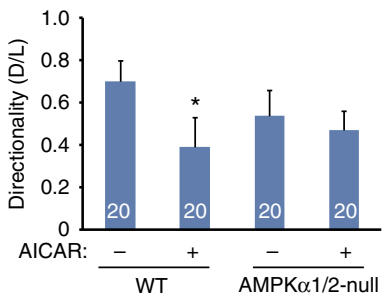

b

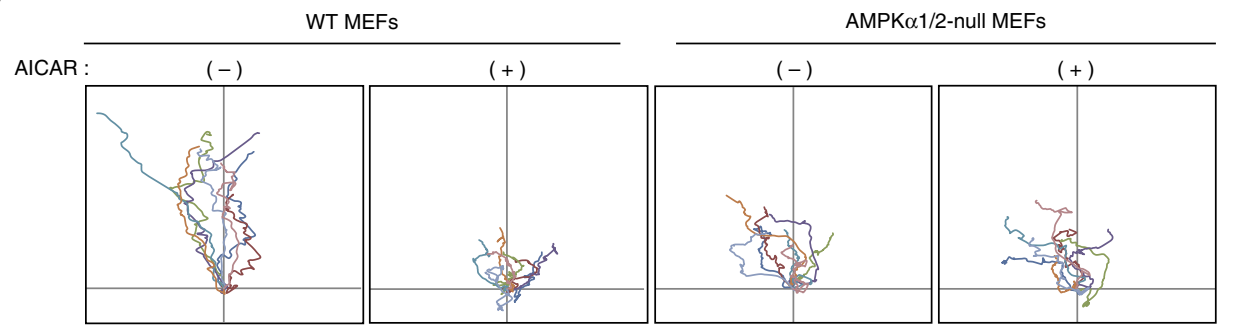

e

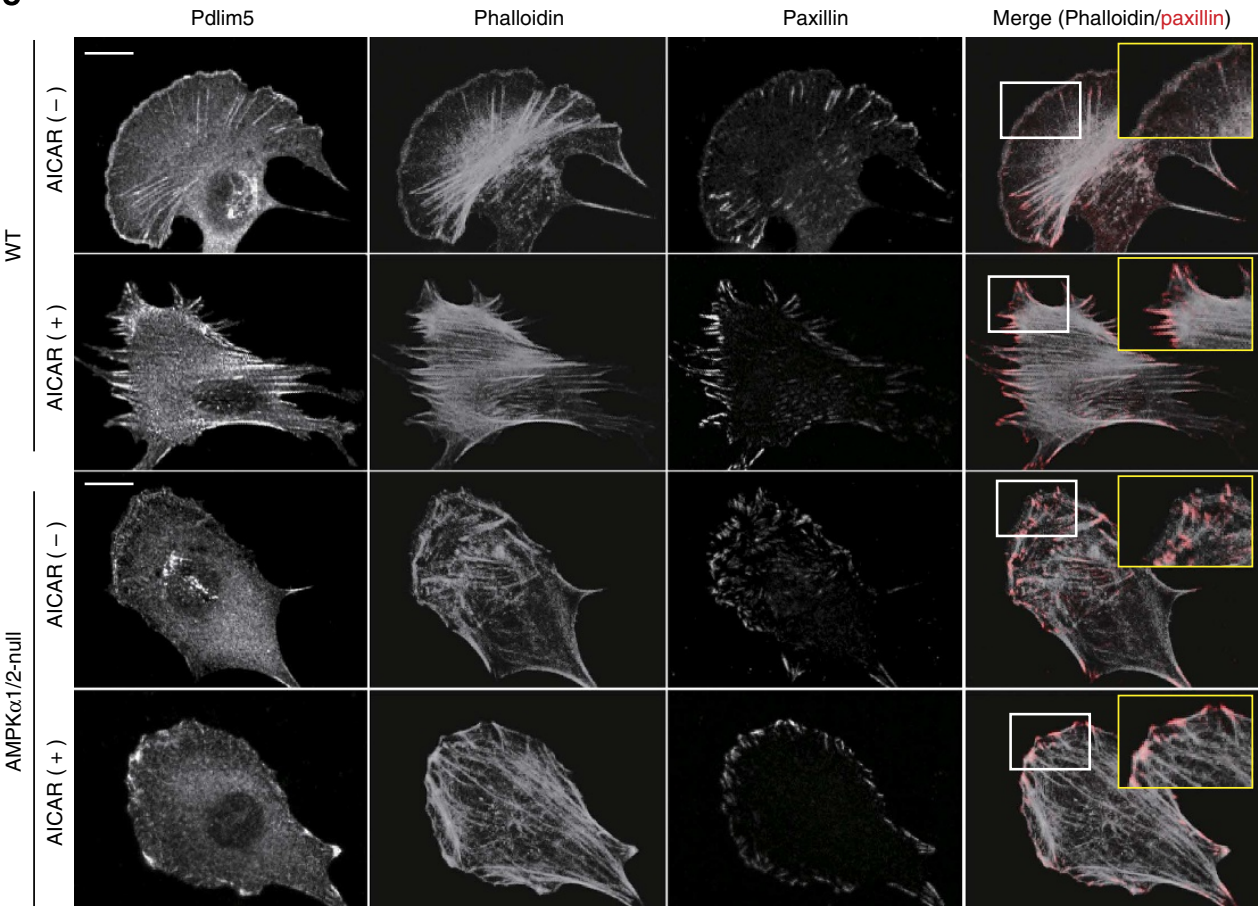

Figure 4 | Pdlim5 phosphorylation was blocked in AMPK $\alpha$ 1/2-null MEFs. (a) WT or AMPK $\alpha 1 / 2-n u l l$ MEFs were stimulated with AICAR (1 mM) for $15 \mathrm{~min}$. TCLs were subjected to immunoblotting with indicated antibodies. (b) Phase-contrast images of WT-MEFs and AMPK $\alpha 1 / 2-$ null MEFs were collected during the scratch assay in the presence or absence of AICAR $(1 \mathrm{mM})$. Each panel shows analysis of migration paths over $8 \mathrm{~h}$. The origins of migration of each cell were superimposed at [0, 0]. (c) Bar graph showing the migrating speed of each cell (from b). (d) Bar graph showing migration directionality of each cell (from $\mathbf{b}$ ). Numbers in the bars indicate $n$. Data are representative of means \pm s.e.m. from three independent experiments.

Significance of differences between series of results was assessed using one-way analysis of variance, followed by a post-hoc comparison with Dunnett's method for multiple comparisons. ${ }^{\star} P<0.01$ compared with WT without AICAR treatment. (e) Immunostained images of WT-MEFs and AMPK $\alpha 1 / 2$-null MEFs in the presence or absence of AICAR $(1 \mathrm{mM})$. These cells were stained with a Pdlim5 antibody, phalloidin and anti-paxillin antibody. Magnified images outlined by yellow squares show the areas outlined by white squares. Scale bar, $10 \mu \mathrm{m}$.

was co-expressed with mCherry instead of being tagged with EGFP (Raichu-Rac1/KDR/Pdlim5-T2A-mCherry) (Fig. 7d). Rac1 activity was lower in cells expressing S177D-Pdlim5 than in those expressing WT-Pdlim5 or S177A-Pdlim5, especially in the cell periphery (Fig. 7e and Supplementary Fig. 14). Thus, these findings indicated that Racl activity was suppressed in cells expressing S177D-Pdlim5, especially in the cell periphery, resulting in dislocation of the Arp $2 / 3$ complex. On the other hand, we observed no changes in RhoA or Cdc42 activity (Fig. 7b,c and Supplementary Fig. 15).

Pdlim5 phosphorylation altered the interaction with Arhgef6. To investigate the molecular mechanism by which S177D-Pdlim5 suppresses Racl activity, we performed GST pull-down assays followed by high-sensitivity shotgun liquid chromatography-mass 
a

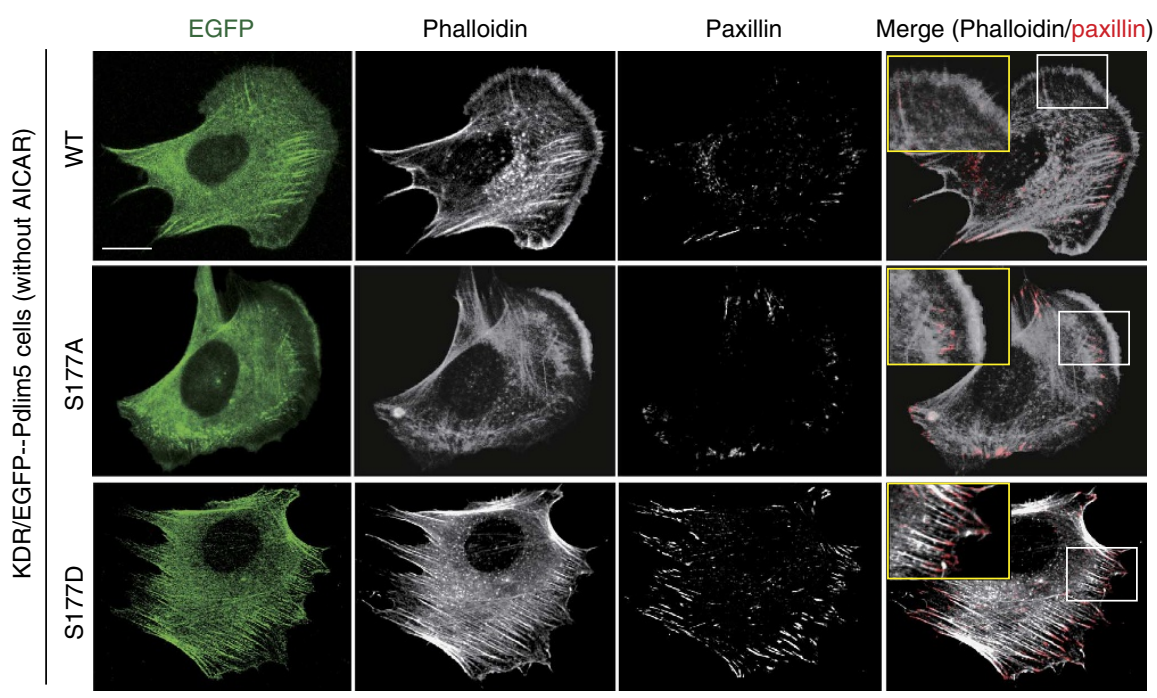

b

KDR/EGFP-WT-Pdlim5 cell (before and after AICAR treatment)
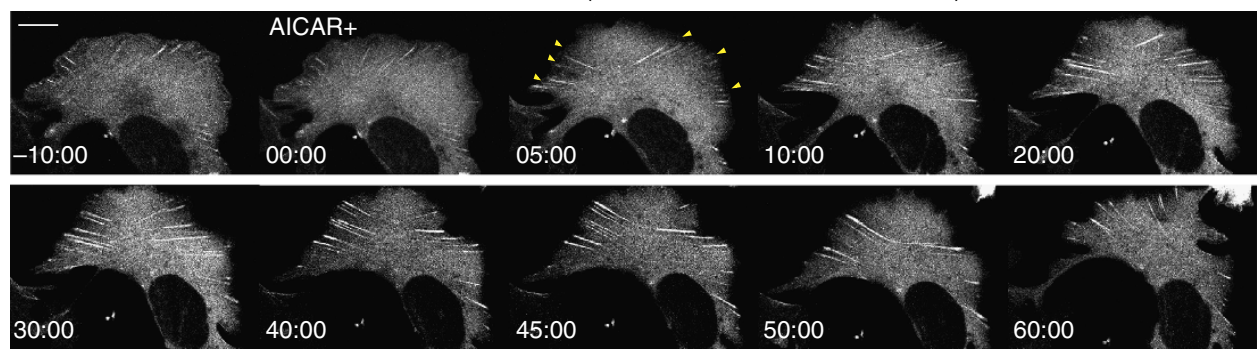

C

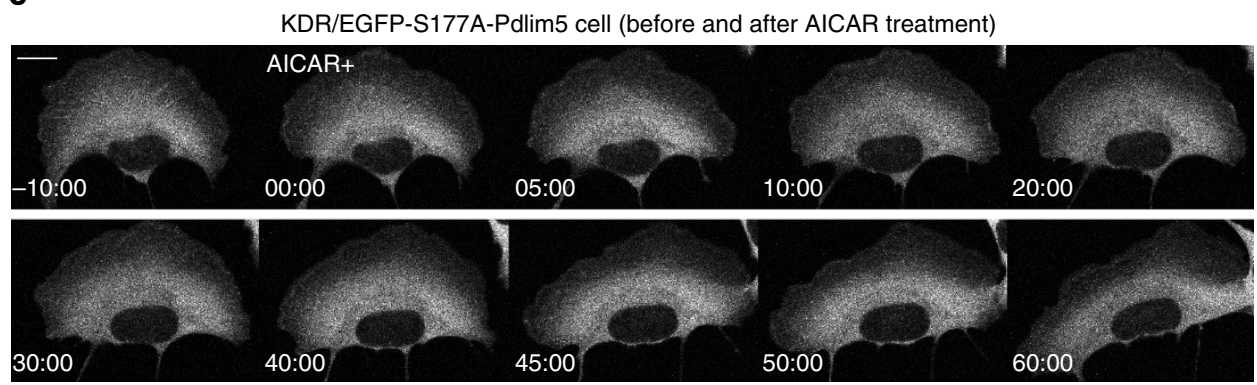

Figure 5 | Ser177 phosphorylation of Pdlim5 causes reorganization of lamellipodia, stress fibres and focal adhesions. (a) GFP images (left) and immunostained images of KDR/EGFP-Pdlim5 cells (WT, S177A and S177D). Cells were stained with phalloidin and anti-paxillin antibody, to visualize actin microfilaments and focal adhesions, respectively. Magnified images outlined by yellow squares show the area outlined by the white squares. Scale bar, $10 \mu \mathrm{m}$. (b,c) Sequential GFP images of KDR/EGFP-WT-Pdlim5 (b) or KDR/EGFP-S177A-Pdlim5 (c) cells before and after AICAR stimulation (2 mM). Yellow arrowheads represent dorsal stress fibres growing from the opposite side after AICAR treatment of KDR/EGFP-WT-Pdlim5 cells. Scale bar, $10 \mu \mathrm{m}$.

spectrometry, using total cell lysates from U937 cells. Among a total of 1,225 proteins detected, several RhoGEFs were more prominently associated with GST-WT-Pdlim5 than with GSTS177D-Pdlim5 (Supplementary Table 1). Among them, we focused on Arhgef6, because it exclusively associated with WT-Pdlim5 (Fig. 8a) and can activate Racl at the leading edge of migrating cells $^{22,23}$. Both the biochemical dissociation of Arhgef6 and S177DPdlim5, and the intracellular co-localization of Arhgef6 with Pdlim5 at the cell periphery were disrupted in KDR/S177D-Pdlim5 cells (Fig. 8b). These findings suggested that Ser177 phosphorylation of Pdlim5 disrupted the recruitment of Arhgef6 at the cell's leading edge, potentially suppressing Racl activity. Next, we compared the effect of Arhgef6 knockdown on cell migration and morphology with that of Ser177 phosphorylation of Pdlim5 (Fig. 8c and Supplementary Fig. 16). The similarity was only partial: Arhgef6-knockdown vSMCs exhibited a disturbed migration relative to control vSMCs (Supplementary Fig. 16b-d) and defective lamellipodia (Fig. 8c), but not elevated formation of stress fibres (Fig. 8c).

Pdlim5 recruits AMPK onto actin filaments. To investigate how AMPK signalling is transmitted to peripheral actin filaments, we examined the physical link between actin filaments, Pdlim5 and AMPK. Immunoprecipitation/immunoblotting of HEK293T cells co-transfected with V5-tagged AMPK $\alpha$ and FLAG-tagged Pdlim5 (WT, $\triangle \mathrm{PDZ}$ or $\triangle \mathrm{LIM}$ ) demonstrated that AMPK bound to Pdlim5 through the LIM domain (Fig. 9a). Next, we performed an F-actin-binding assay, in which F-actin and its binding proteins are found in the pellet fraction, to determine whether Pdlim5 
a

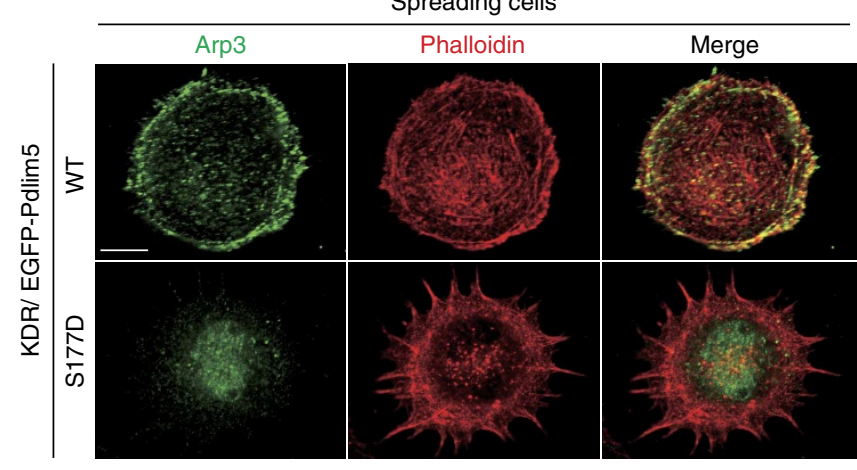

b

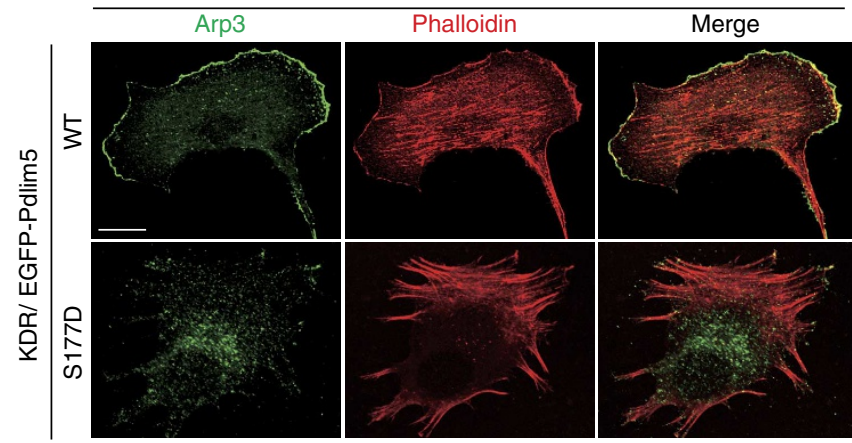

Figure 6 | Ser177 phosphorylation of Pdlim5 leads to altered Arp2/3 complex localization. $(\mathbf{a}, \mathbf{b})$ Immunostaining of spreading (a) or polarized (b) KDR/EGFP-Pdlim5 cells. Cells were fixed and stained with an Arp3 antibody and phalloidin. Scale bars, $10 \mu \mathrm{m}$.

promotes the recruitment of AMPK onto actin filaments. In the absence of Pdlim5, AMPK was found exclusively in the supernatant (Fig. 9b). However, in the presence of Pdlim5, AMPK shifted from the supernatant to the pellets and this shift was greatly stimulated by the presence of $\alpha$-actinin (Fig. 9b). These findings indicate that Pdlim5 binds AMPK directly and promotes the recruitment of AMPK onto F-actin, a process mediated by $\alpha$-actinin.

\section{Discussion}

The results described here reveal the mechanism by which augmented AMPK activity inhibits cell migration. In this study, we serendipitously identified Pdlim5 as a novel substrate of AMPK. On augmentation of AMPK activity, Pdlim5 was phosphorylated at Ser177, disrupting its association with Arhgef6 at the cell periphery and suppressing Racl activity at the leading edge of cell. Suppression of Racl activity dislocated the Arp2/3 complex from the leading edge, resulting in attenuation of lamellipodia formation and inhibition of cell migration.

We previously established a unique screening method using two-step column chromatography combined with an in vitro kinase reaction. Using this method, we identified CLIP-170 as a novel AMPK substrate ${ }^{4}$. In this study, we identified Pdlim5 as another substrate of AMPK by probing cells with a pACC antibody after AMPK activation. The amino-acid sequence surrounding Ser177 of Pdlim5 is very similar to a sequence in ACC, which may explain why we were able to discover this new AMPK substrate using a pACC antibody. Importantly, Pdlim5 is not phosphorylated at all before stimulation with AMPK activators, whereas other AMPK substrates such as ACC and
CLIP-170 (ref. 4) are already phosphorylated to some extent even before stimulation. These findings suggest that Pdlim5 is phosphorylated only under stressed conditions in which AMPK activity is augmented. Accordingly, we conclude that the functional impact of Pdlim5 phosphorylation by AMPK is exerted only under cellular conditions associated with augmented AMPK activity. Thus, Pdlim5 phosphorylation might cause different biological effects than phosphorylation of other AMPK substrates.

In mouse, the double knockout of AMPK $\alpha 1 \alpha 2\left(\mathrm{AMPK} \alpha 1^{-1-}\right.$ $\alpha 2^{-/-}$) is early embryonic lethal ${ }^{24}$, but AMPK $\alpha$-null MEFs are viable $^{25}$. AMPK-null cells exhibit a metabolic shift towards aerobic glycolysis ${ }^{26}$, as well as abnormalities in cell polarity and cellular structures ${ }^{2}$. Pharmacological inhibition of AMPK also perturbs cell polarity and migration ${ }^{4}$. Thus, it is reasonable to conclude that AMPK activity affects directional cell migration by regulating cell polarity. However, as AMPK has many substrates other than Pdlim5, it is still difficult to infer the role of Pdlim5 phosphorylation from the lethal phenotype of the AMPK $\alpha 1^{-I-} \alpha 2^{-I-}$ mouse. On the other hand, two studies have reported different phenotypes of Pdlim5 homozygous knockout mice: dilated cardiomyopathy ${ }^{27}$ and embryonic lethality probably due to embryonic heart/circulation failure ${ }^{28}$. Pdlim5 associates with the actin cytoskeleton and promotes the assembly of protein complexes by acting as a scaffold protein, and thus recruit proteins that modulate cell architecture, actin dynamics and signal transduction ${ }^{19}$ to the actin filaments. Therefore, both phenotypes of Pdlim5 homozygous knockout mice may be attributed to the loss of organized cytoskeletal structures due to progressive loss of protein complex components. Similarly, we postulate that Pdlim5 and its phosphorylation play an important role in regulation of cell morphology and migration, although no previous reports have described the cell migratory behaviour of Pdlim5-knockout cells.

In this study, we focused on determining how AMPK-induced Ser177 phosphorylation of Pdlim5 inhibits cell migration. We found that Pdlim5 localized on actin-filament structures such as stress fibres and focal adhesions, as well as at the cell cortex (including lamellipodia), and that Ser177 phosphorylation itself did not influence Pdlim5 localization. However, the phosphomimetic mutant S177D-Pdlim5 caused characteristic morphological changes such as defective lamellipodia, enhanced ventral stress fibres and displacement of focal adhesions to the cell edge. These specific morphological changes at the cell edge were also observed on pharmacological activation of endogenous AMPK. Thus, the major initial morphological changes resulting from Ser177 phosphorylation of Pdlim5 seemed to arise from the cell cortex. Arhgef6 belongs to the Dbl family of GEFs, defined by the presence of tandem Dbl homology and Pleckstrin homology domains, and functions as a Rac-specific GEF at the cell periphery $22,23,29$. Arhgef6 is recruited to a signalling complex consisting of integrin-linked kinase, particularly interesting cysteine-histidine-rich protein and parvin (IPP complex) by binding to parvin ${ }^{30,31}$ (Fig. 10), which plays an important role in cell spreading and motility ${ }^{32}$, and may be involved in the Pdlim5 phosphorylation signal mediated by Arhgef6. The IPP complex assembles at small focal complexes at the tips of lamellipodia of migrating cells and then interacts with the cytoplasmic tails of $\beta$-integrin molecules to connect them to the actin cytoskeleton ${ }^{32}$. Thus, Arhgef6 activates Racl and reorganizes the actin cytoskeleton around the focal complex of lamellipodia, which is necessary for cell spreading and migration ${ }^{29}$. Moreover, $\alpha$-actinin also binds to parvin directly ${ }^{33}$ and is involved in the IPP complex $^{31}$. As Pdlim 5 binds to $\alpha$-actinin directly ${ }^{34}$, it is likely to be that Pdlim5 is recruited to the IPP complex in close proximity to Arhgef6 via binding to $\alpha$-actinin. It is particularly noteworthy 


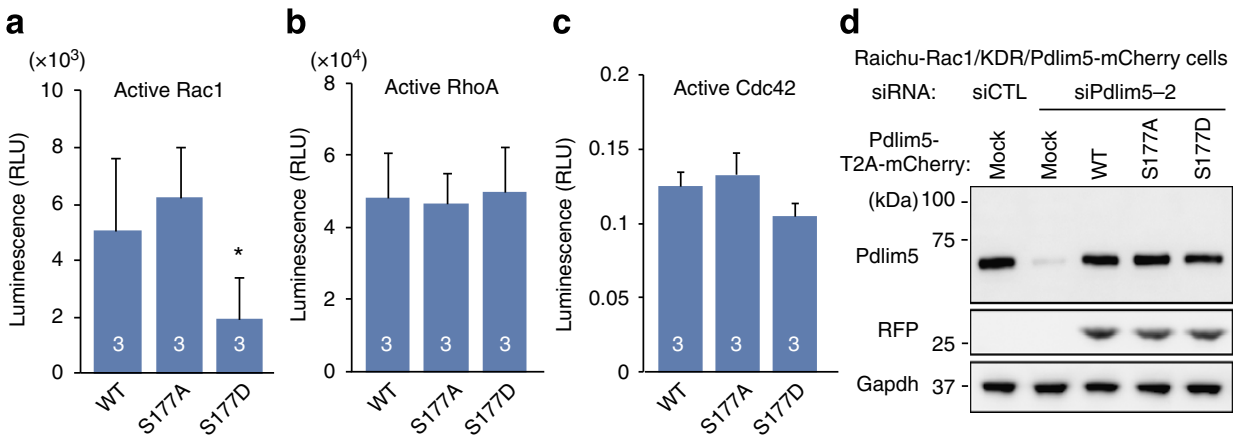

e

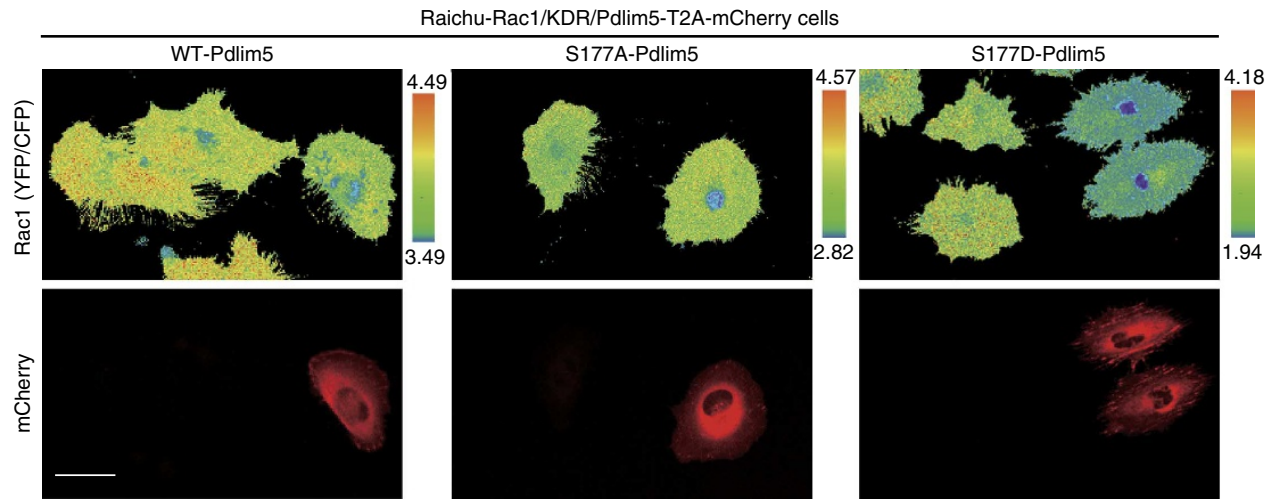

Figure 7 | Ser177 phosphorylation of Pdlim5 suppressed Rac1 activity. (a-c) Activities of Rac1 (a), RhoA (b) and Cdc42 (c) in KDR cells were quantitated using G-LISA specific for Rac1, RhoA and Cdc42, respectively. Numbers in the bars indicate $n$. Data are representative of means \pm s.e.m. from three independent experiments. Significance of differences between series of results was assessed using one-way analysis of variance, followed by a post-hoc comparison with Dunnett's method for multiple comparisons. ${ }^{\star} P<0.01$ compared with KDR/EGFP-WT-Pdlim5. (d) The KDR system was established in vSMCs stably expressing FRET probes specific for Rac1 (Raichu-Rac1/vSMCs). Raichu-Rac1/vSMCs were transfected with either siCTL or siPdlim5-2. siPdlim5-2-resistant Pdlim5-T2A-mCherry (WT, S177A and S177D) was introduced via adenoviral-mediated gene delivery (Raichu-Rac1/KDR-Pdlim5T2A-mCherry cells). TCLs were subjected to immunoblotting. (e) Imaging of Rac1 activity. Each type of Raichu-Rac1/KDR-Pdlim5-T2A-mCherry cell (WT, S177A and S177D) was imaged for YFP and CFP. FRET efficiencies are shown as YFP/CFP ratio images. Scale bars, $20 \mu \mathrm{m}$.

that S177D-Pdlim5 disrupted the physical association between Pdlim5 and displaced Arhgef6 from the cell periphery (Fig. 8). These findings led us to speculate that Arhgef6 is displaced from the IPP complex on Ser177 phosphorylation of Pdlim5 by AMPK, resulting in the suppression of Racl activity at the cell's leading edge. Racl is predominantly localized at the plasma membrane ${ }^{35}$ and GTP-bound active Racl at the cell periphery can activate Arp2/3 complex through recruitment of the Wiskott-Aldrich Syndrome protein family verprolin homologous complex ${ }^{10,11}$. Therefore, we expected that the function of Arp $2 / 3$ complex would also be suppressed in KDR/S177D-Pdlim 5 cells. In fact, the Arp $2 / 3$ complex was displaced from the cell periphery and distributed throughout the cytoplasm in KDR/S177D-Pdlim5 cells, consistent with a previous study demonstrating that inhibition of Rac1 activity interferes with intracellular localization of the Arp2/3 complex ${ }^{36}$. Furthermore, the morphological characteristics observed in KDR/S177D-Pdlim5 cells were quite similar to those in cells with a functional defect in the Arp2/3 complex ${ }^{37-39}$. As Arp2/3 complexes play a pivotal role in organizing branched actin filament networks to form lamellipodia 10,11 , the attenuation of lamellipodia formation and inhibition of cell migration observed in KDR/S177D-Pdlim5 cells was plausible. Thus, our findings strongly suggest that Ser177 phosphorylation of Pdlim5 by AMPK suppresses Rac1 activity by displacing Afhgef6 from the cell periphery, leading to functional suppression of the Arp2/3 complex. However, as the morphological phenotype of Arhgef6-knockdown cells was not entirely consistent with that of KDR/S177D-Pdlim5 cells, we must consider the idea that mechanisms other than the Arhgef6Rac1-Arp2/3 complex pathway contribute to the phenotypes resulting from Ser177 phosphorylation of Pdlim5. One possible mechanism involves a relative increase in RhoA activity over Racl due to a reduction in Racl activity, which may contribute to the phenotype even in the absence of elevated RhoA activity. Consistent with this idea, the relative balance between Racl and RhoA activities regulates cell morphology and migratory behaviour ${ }^{40,41}$. Another possibility is that other GEFs or GTPase-activating proteins contribute to the phenotype of KDR/S177D-Pdlim5 cells. Indeed, dedicator of cytokinesis 2 is also a Rac-specific GEF and interacted with WT-Pdlim5 more strongly than with S177D-Pdlim5 (Supplementary Table 1).

Another important question relates to the mechanism underlying excessive formation of stress fibres, observed in both vSMCs treated with AMPK activator and KDR/S177D-Pdlim5 cells. In particular, cells stimulated with AMPK activators exhibited striking elongation of dorsal stress fibres from the cell periphery. This finding was in excellent agreement with a previous report demonstrating that Arp2/3-defective cells exhibited a higher growth rate of dorsal stress fibres ${ }^{39}$. The authors of that report proposed that the elevated growth of dorsal stress fibres may result from the increased concentration of cytoplasmic G-actin caused by the absence of Arp2/3-nucleated barbed ends ${ }^{10}$. In addition, $\mathrm{mDia}$ remained localized at the cell periphery in KDR/ S177D-Pdlim5 cells, whereas Arp2/3 complex moved from the periphery to the cytosol. mDia is another major actin filament nucleator that nucleates linear actin filament at the cell periphery 
a

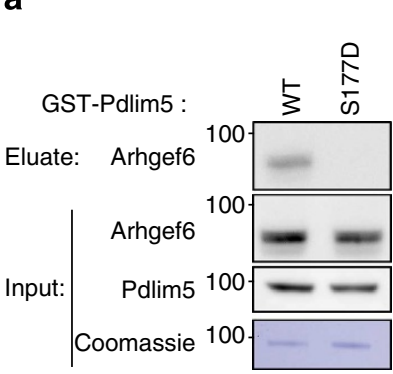

b
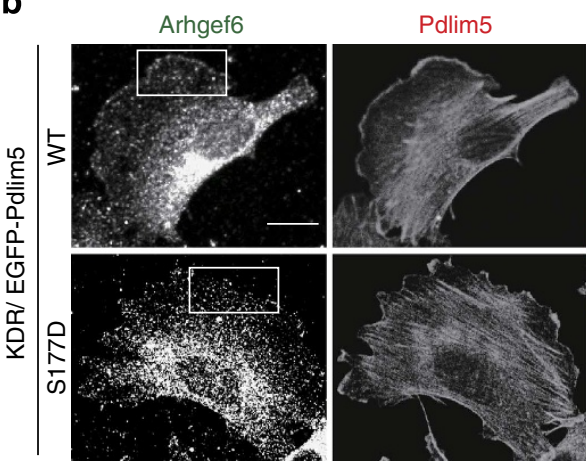

Merge (Arhgef6/Pdlim5)

C

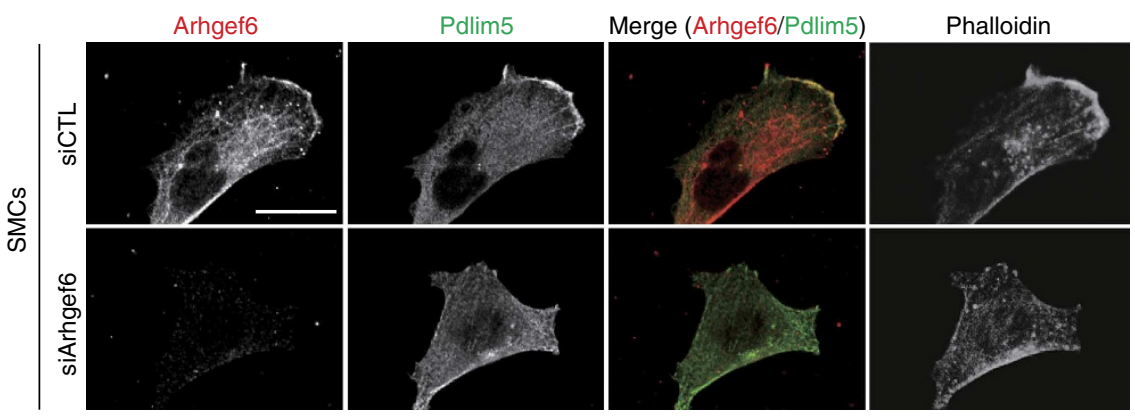

Figure 8 | Ser177 phosphorylation of Pdlim5 disrupts its association with Arhgef6 at the cell periphery. (a) Immunoblotting analysis of GST-Pdlim5 pull-down assay. Eluates were subjected to immunoblotting with anti-Arhgef6 antibody. Coomassie staining demonstrates equal loading of GST-Pdlim5 proteins. (b) Immunostaining images of Arhgef6 and Pdlim5 from KDR/EGFP-Pdlim5 cells. Boxed area in KDR/EGFP-WT-Pdlim5 cell highlights representative co-localization of Arhgef6 with Pdlim5 at the cell periphery. Scale bars, $10 \mu \mathrm{m}$. (c) Immunostained images of Arhgef6 knockdown vSMCs stained with anti-Pdlim5 antibody, anti-Arhgef6 antibody and phalloidin. Scale bars, $10 \mu \mathrm{m}$.

a

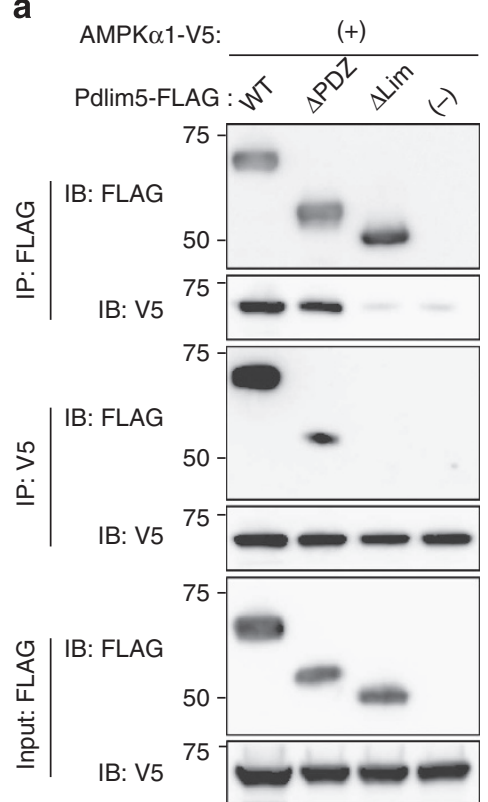

b

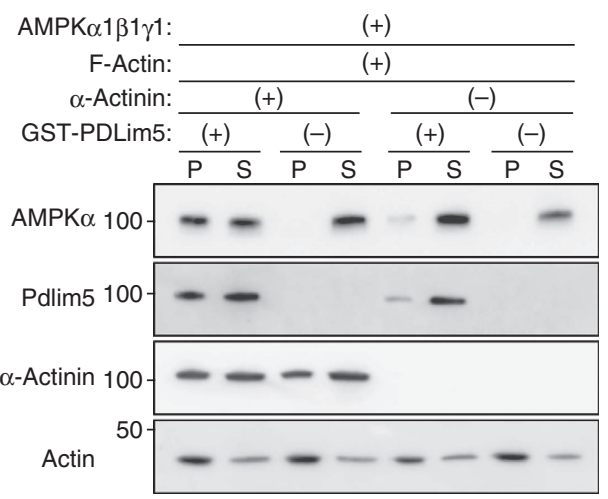

Figure 9 | AMPK is recruited onto F-actin by directly binding the LIM domain of Pdlim5. (a) HEK293T cells were co-transfected with V5-tagged AMPK $\alpha$ and FLAG-tagged Pdlim5 (WT, $\triangle \mathrm{PDZ}$ or $\triangle$ LIM domain). TCLs were immunoprecipitated by FLAG or V5 and then immunoblotted with the indicated antibodies. (b) F-actin binding assay of AMPK. AMPK was mixed with a fixed amount of F-actin in the presence or absence of $\alpha$-actinin and GST-Pdlim5, incubated for $1 \mathrm{~h}$ at $24^{\circ} \mathrm{C}$ and then centrifuged at $150,000 \mathrm{~g}$ for $1.5 \mathrm{~h}$ at $24^{\circ} \mathrm{C}$, to pellet the F-actin polymer and associated proteins. A sample of the pellet $(\mathrm{P})$ and supernatant $(\mathrm{S})$ were analysed by immunoblotting for the indicated antibodies.

and can elongate actin directly proportional to the G-actin monomer concentration ${ }^{42}$. Thus, $\mathrm{mDia}$ at the cell periphery under increased G-actin concentration may also contribute to elevated formation of stress fibres and filopodia in KDR/S177DPdlim5 cells. Taken together, these data indicate that the morphological and migratory phenotypes observed in cells 

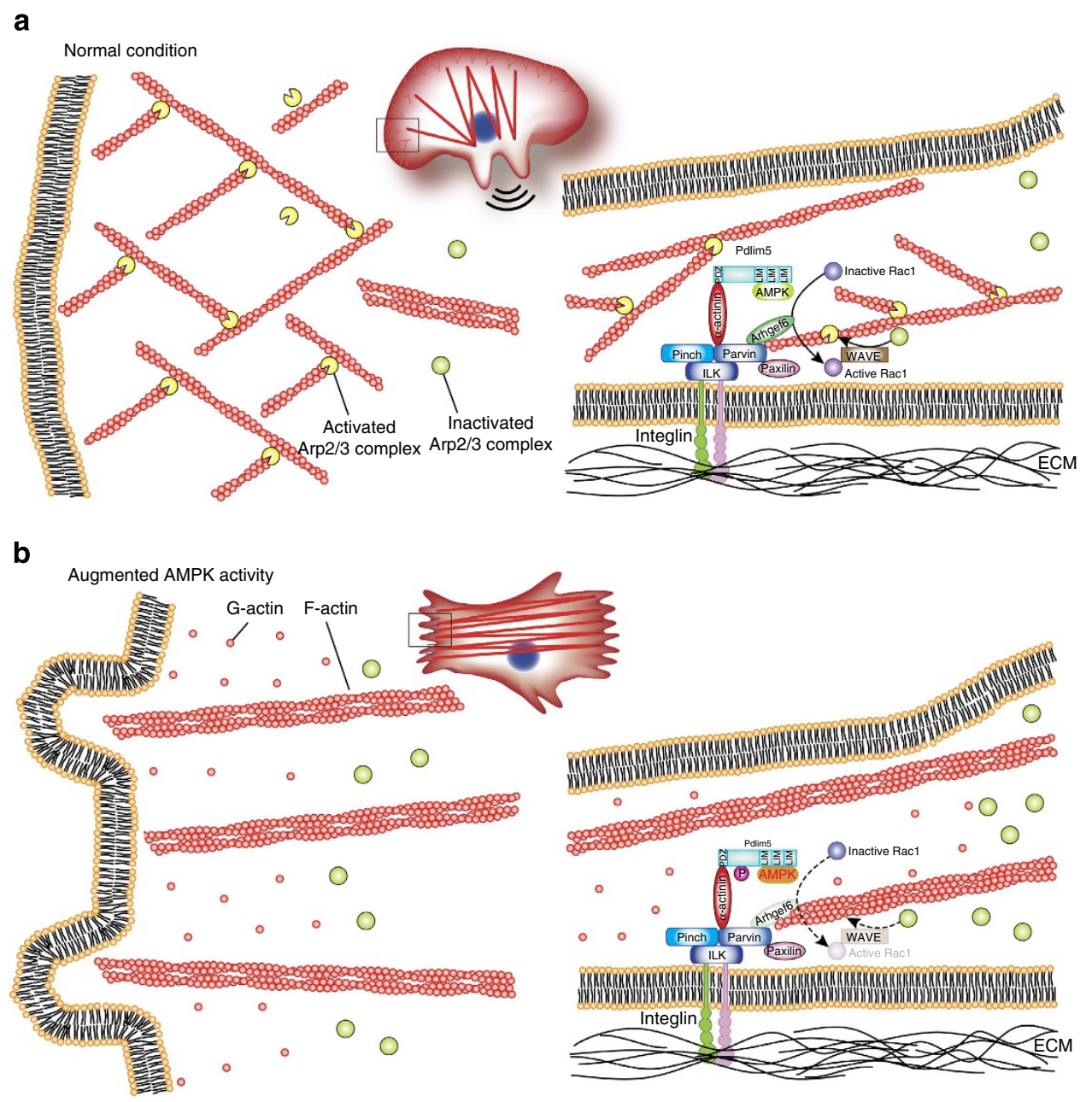

Figure 10 | Model depicting how the Ser177 phosphorylation of Pdlim5 attenuates lamellipodia formation. (a) Under normal conditions, Arhgef6 recruited to the IPP complex at the cell periphery activates Rac1, contributing to efficient lamellipodia formation. (b) Once AMPK activity is augmented, Ser177 of Pdlim5 is phosphorylated, displacing Arhgef6 from the IPP complex and causing attenuation of lamellipodia. Boxed areas at the cell periphery are expanded, representing views from the top (left) and from the side (right); see text for explanation. ILK, integrin-linked kinase; PINCH, particularly interesting new cysteine-histidine-rich protein; Pdlim5, PDZ and LIM domain 5; WAVE, Wiskott-Aldrich Syndrome protein family verprolin homologous.

expressing Ser177-phosphorylated Pdlim5 might be caused not only by the Arhgef6-Rac1-Arp2/3 complex pathway, but also by other additional mechanisms/pathways including other GEFs/ GTPase-activating proteins, other Rho-GTPases (RhoA and Cdc42) and mDia. In addition, the data in Supplementary Fig. 6 demonstrate that KDR/S177A-Pdlim5 cells moved more slowly when treated with AMPK activator, indicating that Pdlim5 is the primary regulator of migration downstream of AMPK, although other pathways are also involved.

We observed a physical and functional association between AMPK, Pdlim5 and F-actin. Pdlim5 binds to AMPK directly through its LIM domain and to F-actin through its PDZ domain, which places AMPK close to F-actin. This interaction seems to rapidly and efficiently transmit AMPK signalling to the actin filament architecture. Thus, we propose that once energy depletion occurs and AMPK is activated, the signal might be transmitted to peripheral actin filaments through this complex, leading to the remodelling of actin-filament architecture and inhibition of cell migration.

A striking feature of the regulation of cell migration by AMPK activity level is that both suppression and augmentation of AMPK inhibit cell migration. Cell migration is a highly complex behaviour that is accomplished by tightly orchestrated dynamic remodelling of the actin cytoskeleton and microtubule network ${ }^{16}$ We previously reported that suppression of AMPK activity inhibits cell migration by hyperstabilizing the microtubule cytoskeleton via dephosphorylation of the microtubule plus-end protein CLIP-170 (ref. 4). In this study, we demonstrated that augmentation of AMPK activity inhibits cell migration by reorganizing actin filaments through phosphorylation of Pdlim5. Thus, the effects of high or low AMPK activity on cell migration may be mediated by completely different mechanisms acting on different substrates, and the two types of substrates may regulate cell migration separately by sensing the level of AMPK activity between two extremes.

\section{Methods}

Reagents and antibodies. The following reagents were purchased from the indicated suppliers: AICAR (Sigma-Aldrich), 2-DG (Sigma-Aldrich), A769662 (Santa Cruz Biotechnology) and GST-AMPK $\alpha 1 / \beta 1 / \gamma 1$ (Carna Biosciences). The following antibodies were purchased from the indicated suppliers: anti-AMPK $\alpha$ (1:2,000; Cell Signaling, 2603), phospho-Thr172 AMPK $\alpha$ (1:2,000; Cell Signaling, 2535), ACC (1:2,000; Cell Signaling, 3676), phospho-Ser79 ACC (1:2,000; Cell Signaling, 3661), anti-paxillin (1:1,000, Zymed Laboratories), anti- $\alpha$-actinin (D6F6) (1:1,000 for immunoblot; Cell Signaling, 6487), monoclonal anti- $\alpha$-actinin (1:1,000 for immunostain; Sigma-Aldrich, A5044), anti-Arp2 antibody (1:1,000; Cell Signaling, 3128), anti-Arp3 (FMS338) (1:500 for immunostaining; Abcam, ab49671), 
anti-Arpc2 (EPR8533) (1:2,000; Abcam, ab133315), anti-Arhgef6/Cool2/ $\alpha$ PIX (C23D2) (1:1,000 for immunoblot; 1:400 for immunostain; Cell Signaling, 4573), anti-Gapdh antibody (1:5,000; Millipore, MAB374), anti-GFP-horseradish peroxidase (HRP) (1:3,000; MBL, 598-7), anti-RFP-HRP (1:3,000; MBL, PM005-7), antiFLAG M2-HRP (1:5,000; Sigma-Aldrich, A8592), anti-V5-HRP antibody (1:5,000; Life Technologies, R961-25), HRP-coupled goat anti-rabbit (1:8,000; Cappel, 55696), anti-mouse IgG (1:8,000; Cappel, 55550), Alexa Fluor 488- (1:1,000 for staining; Life Technologies, A11029), Alexa Fluor 546- (1:1,000 for staining; Life Technologies, A11003) and Alexa Fluor 568-labelled secondary antibodies (1:1,000 for staining; Life Technologies, A11011), and Alexa Fluor 647 phalloidin (1:100 for staining; Cell Signaling, 8940). Anti-FLAG M2 affinity gel (A2220) and anti-V5 agarose affinity gel (A7345) were from Sigma-Aldrich. We used three different AMPK activators: AICAR is metabolized intracellularly to ZMP (5-aminoimidazole-4-carboxamide-1- $\beta$-D-ribofuranotide), an AMP analogue. A769662 is a thienopyridone derivative that acts as an allosteric activator of the AMPK by binding to an alternative site that does not overlap with the AMP-binding site.

2-DG is a non-metabolizable glucose analogue and inhibitor of phosphohexose isomerase that inhibits glycolysis and mimics glucose starvation, increases the AMP/ATP ratio and thereby activates AMPK.

Antibodies for Pdlim5 and pS177 of Pdlim5. Six polyclonal Pdlim5 antibodies (1:1,000 for immunoblotting and immunostaining) and two polyclonal phospho-Ser177 (pS177)-Pdlim5 antibodies (1:1,000 for immunoblotting) were generated as follows. Three different peptides corresponding to mouse Pdlim5 sequences (amino acids 229-245, QGDIKQQNGPPRKHIVEC; amino acids 290-306, CTGTEHLTESENDNTKKA; and amino acids 381-397, SSGTGASVG PPQPSDQDC), as well as Ser-phosphorylated and non-phosphorylated peptides corresponding to the mouse Pdlim5 sequences surrounding Ser177 (amino acids 172-182, LHLSA(pS)GLHVS), were chemically synthesized. Rabbits were immunized five times with the keyhole limpet haemocyanin-phosphopeptide conjugates mixed with Freund's complete adjuvant and bled 7 days after the last immunization. Phosphopeptide-reactive antibody was captured by a column containing phosphopeptide-conjugated Sepharose. The antibodies were then eluted, and those reactive to sequences other than phosphoserine were removed using a column containing non-phosphorylated peptides. Specific reactivity with the targeted phosphoserine sequence was confirmed by ELISA using phosphorylated and non-phosphorylated peptides.

Cell culture and siRNA transfection. C2C12 cells (an immortalized mouse myoblast cell line) and HEK293T cells were obtained from the American Type Culture Collection. A vSMC line established from thoracic aorta of a p53-knockout mice (P53LMACO1) was purchased from Health Science Research Resources Bank. These cells were maintained in DMEM medium (Sigma-Aldrich) supplemented with 10\% FCS (Equitech-Bio) and 1\% penicillin-streptomycin at $37^{\circ} \mathrm{C}$ in a $5 \% \mathrm{CO}_{2}$ atmosphere at constant humidity and passaged by trypsinization at $70-80 \%$ confluence. HL60 cells and RAW264.7 cells were obtained from the Japanese Collection of Research Bioresources Cell Bank and American Type Culture Collection, respectively. These cells were maintained in RPMI1640 medium with 10\% FCS and 1\% penicillin-streptomycin. HEK293T cells and vSMCs were transfected with plasmids using Lipofectamine 2000 reagent (Invitrogen). To knock down endogenous Pdlim5, C2C12 cells and vSMCs were transfected with siRNAs $\left(30 \mathrm{nM}\right.$ ) targeting Pdlim5 (siPdlim5-1, sense: $5^{\prime}$ ggaacaauaugucguggauTT-3'; antisense: $5^{\prime}$-auccacgacauauuguuccTT-3'; siPdlim5-2, sense: $5^{\prime}$-ggguaguagcuaugagaauTT- $3^{\prime}$; antisense: $5^{\prime}$-auucucauagcuacuacccTT- $3^{\prime}$ ) using Lipofectamine RNAiMAX (Invitrogen). To knock down endogenous Arpc2, vSMCs were transfected with siRNAs $(5 \mathrm{nM})$ targeting Arpc2 (siArpc2-1, sense: $5^{\prime}$-ggccuauauucauacacgaTT-3'; antisense: $5^{\prime}$-ucguguaugaauauaggccTT-3'; siArpc22 , sense: $5^{\prime}$-gaaccaggauauaauguuuTT- $3^{\prime}$; antisense: $5^{\prime}$-aaacauuauauccugguucTG- $3^{\prime}$ ) using Lipofectamine RNAiMAX (Invitrogen). RAW264.7 cells (mouse leukemic monocyte macrophage cell) were used to check the effect of siRNAs against Arhgef6. RAW264.7 cells were transfected with siRNAs ( $50 \mathrm{nM}$ ) targeting Arhgef6 (siArhgef6-1, sense: $5^{\prime}$-gauucuuaaggugaucgaaTT- $3^{\prime}$; antisense: $5^{\prime}$ -

uucgaucaccuuaagaaucTG-3'; siArhgef6-2, sense: $5^{\prime}$-gugaugaucuagaacgauuTT-3'; antisense: $5^{\prime}$-aaucguucuagaucaucacTG- $3^{\prime}$ ) using GenMute siRNA Transfection Reagent for RAW 264.7 (SignaGen). siControl was used as a negative control. Efficiency of siRNA-mediated knockdown was confirmed at 24 and $72 \mathrm{~h}$ after incubation with siRNAs. AMPK $\alpha$ double-knockout (AMPK $\alpha 1^{-/-} \alpha 2^{-1-}$ ) MEFs $\left(\mathrm{AMPK} \alpha\right.$-null MEFs) ${ }^{25}$ were kindly provided by $\mathrm{Dr}$ B. Viollet (INSERM, France) and maintained in DMEM supplemented with $10 \%$ FCS, $1 \mathrm{mM}$ sodium pyruvate and $1 \%$ penicillin-streptomycin at $37^{\circ} \mathrm{C}$ in a $5 \% \mathrm{CO}_{2}$ atmosphere at constant humidity.

Hypoxia. Cultured cells were exposed to hypoxia for $2 \mathrm{~h}$. Hypoxic conditions ( $1 \%$ $\mathrm{O}_{2}$ ) were maintained in a MCO-5M multi-gas incubator (Sanyo).

Establishment of the KDR system. To replace endogenous Pdlim5 by EGFPtagged recombinant Pdlim5 (WT, S177A or S177D), vSMCs were transfected with siPdlim5-2 to deplete endogenous Pdlim5. Next, $12 \mathrm{~h}$ after siPdlim5-2 transfection, siPdlim5-2-resistant EGFP-tagged Pdlim5 (WT, S177A or S177D) was adenovirally transduced into vSMCs, to establish the KDR system. vSMCs were incubated with adenovirus at a multiplicity of infection of 10 in DMEM supplemented with $10 \%$
FCS at $37^{\circ} \mathrm{C}$ under $5 \% \mathrm{CO}_{2}$ for $30 \mathrm{~min}$, with gentle mixing every $10 \mathrm{~min}$, and then further incubated for $48 \mathrm{~h}$ before analysis.

Purification and identification of Pdlim5. C2C12 cells seeded on 15- $\mathrm{cm}$ dishes $\left(2 \times 10^{6}\right.$ cells per dish) were treated with $2 \mathrm{mM}$ of AICAR for $1 \mathrm{~h}$, harvested and lysed on ice in lysis buffer A (20 mM Tris pH 8.0, 0.5\% NP-40, 0.5\% CHAPS, 20\% acetonitrile, $25 \mathrm{mM} \beta$-glycerophosphate, $10 \mathrm{mM} \mathrm{NaF}$ and protease inhibitor cocktail (Nacalai Tesque)). Lysates were incubated at $4{ }^{\circ} \mathrm{C}$ with agitation for $20 \mathrm{~min}$, followed by centrifugation at $10,000 \mathrm{~g}$ for $20 \mathrm{~min}$. Supernatant from six $15-\mathrm{cm}$ dishes was passed through a $0.45-\mu \mathrm{m}$ sterilization filter and loaded onto a TSK-GEL SuperQ-5PW $(7.5 \times 75 \mathrm{~mm}$, TOSOH $)$ anion-exchange column preequilibrated with column buffer A $(20 \mathrm{mM}$ Tris $\mathrm{pH}$ 8.0, $0.5 \%$ CHAPS, $20 \%$ acetonitrile, $25 \mathrm{mM} \beta$-glycerophosphate, $10 \mathrm{mM} \mathrm{NaF})$. After being washed with column buffer $\mathrm{A}$, proteins were eluted with a linear gradient of $\mathrm{NaCl}(0-1 \mathrm{M}$ over $60 \mathrm{~min})$ at a flow rate of $0.5 \mathrm{ml} \mathrm{min}{ }^{-1}$. Fractions $(0.5 \mathrm{ml}$ each) were collected and a $50-\mu \mathrm{l}$ aliquot of each fraction was analysed by immunoblotting with anti-pACC antibody. The corresponding fractions were prepared in the presence of $0.3 \%$ TFA (trifluoroacetic acid), $0.1 \%$ OG ( $n$-octyl- $\beta$-D-thioglucopyranoside) and $20 \%$ acetonitrile, and loaded onto a Protein-R $(4.6 \times 250 \mathrm{~mm}$, Nacalai Tesque) reversephase HPLC column pre-equilibrated with column buffer B $(0.1 \%$ TFA and $0.1 \%$ OG). After being washed with column buffer B, the proteins were eluted with a linear gradient of acetonitrile $(20-80 \%$ over $60 \mathrm{~min})$ at a flow rate of $0.5 \mathrm{ml} \mathrm{min}^{-1}$ Fractions $(0.5 \mathrm{ml}$ each) were collected and a $25-\mu \mathrm{l}$ aliquot of each fraction was analysed by immunoblotting with anti-pACC antibody. The corresponding fractions were again prepared in the presence of $0.3 \%$ TFA, $0.1 \%$ OG and $20 \%$ acetonitrile, and loaded onto a 5Ph-AR-300 $(4.6 \times 250 \mathrm{~mm}$, Nacalai Tesque) reversephase HPLC column pre-equilibrated with column buffer B. After being washed with column buffer B, proteins were eluted with a linear gradient of acetonitrile (20-80\% over $60 \mathrm{~min}$ ) at a flow rate of $0.5 \mathrm{ml} \mathrm{min}^{-1}$. Each fraction was analysed by SDS-PAGE and visualized by silver staining and immunoblotting with antipACC antibody. Target bands matching the pACC antibody cross-reacting bands were excised from the gel and analysed using matrix-assisted laser desorption/ ionization-quadrupole-time-of-flight-tandem mass spectrometry (MALDI-QqTOF MS/MS).

Protein purification. Recombinant FLAG-tagged Pdlim5 proteins were purified as follows: HEK293T cells transfected with pEF-DEST51/cFLAG plasmid encoding WT Pdlim5, S175A Pdlim5 or S177A Pdlim5 were lysed in lysis buffer B $(20 \mathrm{mM}$ MOPS pH 7.5, $0.15 \mathrm{M} \mathrm{NaCl}, 0.5 \%$ CHAPS, $1 \mathrm{mM}$ EDTA, 1 mM dithiothreitol (DTT) and protease inhibitor cocktail) and immunoprecipitated with anti-FLAG M2 agarose (Sigma-Aldrich) at $4{ }^{\circ} \mathrm{C}$ for $30 \mathrm{~min}$. The beads were washed three times with wash buffer (20 mM MOPS pH 7.5, $0.3 \mathrm{M} \mathrm{NaCl}, 0.5 \%$ CHAPS, 1 mM EDTA $1 \mathrm{mM}$ DTT and protease inhibitor cocktail) and eluted with elution buffer $(20 \mathrm{mM}$ MOPS pH 7.5, 0.3 M NaCl, 0.5\% CHAPS, $1 \mathrm{mM}$ DTT and $0.5 \mathrm{mg} \mathrm{ml}^{-1}$ FLAG peptide (Sigma-Aldrich)) at $4{ }^{\circ} \mathrm{C}$ for $30 \mathrm{~min}$. After centrifugation, the supernatants were used as recombinant FLAG-tagged proteins. Recombinant GST-Pdlim5 proteins (WT, S177A, S177D and $\triangle \mathrm{PDZ}$ ) were purified as follows: BL21 chemically competent $E$. coli (Invitrogen) were transformed with pGEX-6P-1-WT Pdlim5, pGEX-6P-1-S177A Pdlim5, pGEX-6P-1-S177D Pdlim5 or pGEX-6P-1- $\Delta$ PDZ Pdlim5, and then induced with $0.5 \mathrm{mM}$ isopropyl- $\beta$-D-thiogalactoside (SigmaAldrich) at $25^{\circ} \mathrm{C}$ for $10 \mathrm{~h}$. The cells were collected by centrifugation and lysed by sonication in PBS containing $5 \mathrm{mM}$ EDTA and protease inhibitor cocktail. After addition of $1 \%$ Triton X-100, the lysates were agitated at $4{ }^{\circ} \mathrm{C}$ for $30 \mathrm{~min}$ and pulled down with glutathione-Sepharose 4 Fast Flow (GE Healthcare) at $4{ }^{\circ} \mathrm{C}$ for $1 \mathrm{~h}$. After being washed three times, the proteins were eluted with $15 \mathrm{mM}$ reduced glutathione and ultrafiltered in elution buffer using a Nanosep 10K Device (Pall Life Science)

Immunoblotting. Immunoblotting was performed using the indicated antibodies Blots were cropped such that at least one marker position is present. Uncropped full scans of the figures are supplied in Supplementary Figs 17-23.

Phosphorylation assay. Phosphorylation assays were carried out at $30^{\circ} \mathrm{C}$ in a reaction volume of $10 \mu \mathrm{l}$ containing Tris- $\mathrm{HCl}(20 \mathrm{mM} \mathrm{pH} 7.4)$, glycerol (10\%), $\mathrm{NaCl}(0.3 \mathrm{mM}), \mathrm{AMP}(0.2 \mathrm{mM}), \mathrm{MgCl} 2(10 \mathrm{mM}), \gamma^{32} \mathrm{P}$ ATP (GE Healthcare BioScience, $10 \mu \mathrm{Ci}, 1.7 \mathrm{pmol})$ and AMPK purified from rat liver $\left(2 \mathrm{ng} \mu \mathrm{l}^{-1}\right)$. Purified mouse Pdlim5-cFLAG proteins or purified recombinant GST-fused mouse Pdlim5 proteins were used as substrates. After $60 \mathrm{~min}$, reactions were terminated and ultrafiltered in PBS containing 0.1\% SDS using a Nanosep 10K Device (Pall Life Science). Each fraction sample was analysed by SDS-PAGE and visualized by autoradiography. For immunoblotting with anti-pS177 antibody, recombinant GST-fused Pdlim5 proteins were incubated under the same conditions, except that ATP $(0.1 \mathrm{mM})$ was included instead of $\gamma^{-}{ }^{32} \mathrm{P}$ ATP.

Scratch assay. KDR/EGFP-Pdlim5 (WT, S177A and S177D) cells or MEFs were plated on a collagen-coated $35-\mathrm{mm}$ glass dish at a density of $5 \times 10^{5} \mathrm{~cm}^{-2}$. Eight hours after plating, a scratch was made with a P-200 pipette tip and the lesions were observed for a total of $8 \mathrm{~h}$. Differential interference contrast images were 
recorded every 5 min using an Olympus LCV110 incubator microscope (Olympus Corporation, Tokyo, Japan). To determine cell trajectories, the centrioles of cell nuclei were tracked throughout time-lapse movies, and migration-tracking images were generated using the MetaMorph 7.1.3.0 software (MDS Analytical Technologies, Downingtown, PA, USA). Overall migration speed was calculated as the average of migration speeds measured every $5 \mathrm{~min}$.

Single-cell migration assay. KDR/EGFP-Pdlim5 (WT, S177A and S177D) cells were plated on a collagen-coated $35-\mathrm{mm}$ glass dish at a density of $5 \times 10^{5} \mathrm{~cm}^{-2}$. Five hours after the plating, we started to observe cell migration by recording differential interference contrast images every $5 \mathrm{~min}$ for a total of $4 \mathrm{~h}$ using an Olympus LCV110 incubator microscope (Olympus Corporation).

Immunocytochemistry and fluorescence imaging. vSMCs or KDR cells were seeded on a collagen-coated 35-mm glass dishes (Asahi Techno Glass Corporation, Chiba, Japan). After cells firmly attached to the dish, they were washed once with warm PBS and fixed with $4 \%$ paraformaldehyde for $5 \mathrm{~min}$ at room temperature. Next, the cells were permeabilized with $0.1 \%$ Triton X-100 in PBS for $5 \mathrm{~min}$ at room temperature and then blocked with $1 \% \mathrm{BSA}$ at $4{ }^{\circ} \mathrm{C}$ overnight. The next day, samples were immunostained with primary antibodies (1:1,000 in 1\% BSA, $1 \mathrm{~h})$. For secondary reactions, species-matched Alexa Fluor 488- or Alexa Fluor 568labelled secondary antibody was used (1:1,000 in 1\% BSA, $30 \mathrm{~min})$. Just before imaging, the sample was incubated with Alexa Fluor 647-conjugated phalloidin in CGS-Sol A for $1 \mathrm{~h}$. Fluorescence images of EGFP, Alexa Fluor 488, Alexa Fluor 546, Alexa Fluor 568 and Alexa Fluor 647 were recorded using an Olympus FV1000-D confocal laser scanning microscope (Olympus Corporation) equipped with a cooled charge-coupled device CoolSNAP-HQ camera (Roper Scientific, Tucson, AZ, USA) and a PLAPO $\times 60$ oil-immersion objective lens. To measure the paxillin-positive area, all intensity profiles were analysed using the MetaMorph 7.1.3.0 software.

Time-lapse imaging of KDR/EGFP-Pdlim5 cells. KDR/EGFP-Pdlim5 (WT and S177A) cells were seeded on collagen-coated $35-\mathrm{mm}$ glass dishes at a density of $4 \times 10^{4} \mathrm{~cm}^{-2}$. Five hours after plating, cells were treated with AICAR $(2 \mathrm{mM})$. The fluorescence images were recorded from $10 \mathrm{~min}$ before to $60 \mathrm{~min}$ after AICAR treatment, using an Olympus IX-81 inverted fluorescence microscope (Olympus Corporation) equipped with a cooled charge-coupled device CoolSNAP-HQ camera (Roper Scientific) and a PLAPO $\times 60$ oil-immersion objective lens controlled by MetaMorph version 7.1.3.0. An EGFP image was obtained every $30 \mathrm{~s}$ through a U-MNIBA2 filter (Olympus Corporation), which had a 470-495 excitation filter and a 510-550 emission filter. Cells were maintained on a microscope at $37^{\circ} \mathrm{C}$ with a $5 \%$ carbon dioxide mixture using a stage-top incubator (Tokai Hit). MetaMorph was used to convert a series of time-lapse images to video format.

Measurement of the GTP-bound form of Rac1, RhoA and Cdc42. KDR cells at $50 \%$ confluence were incubated for $24 \mathrm{~h}$ in FCS-free DMEM to starve the cells, and then treated with $10 \%$ FCS for $30 \mathrm{~min}$. Cell lysates were collected and levels of activated GTP-bound Racl, RhoA and Cdc42 were determined using the G-LISA Rac1 Activation Assay Biochem Kit (BK126, Cytoskeleton Inc., CO, USA), G-LISA RhoA Activation Assay Biochem Kit (BK121, Cytoskeleton Inc.) and G-LISA Cdc42 Activation Assay Biochem Kit (BK127, Cytoskeleton Inc.), respectively.

Imaging of Rho GTPases (Rac1, RhoA and Cdc42) activities. To visualize activities of Rho GTPases in living cells, we established a vSMC cell line stably expressing a Rho GTPase FRET biosensor consisting of cyan fluorescent protein (CFP) and yellow fluorescent protein (YFP), as previously described ${ }^{43}$. In brief, vSMCs were transfected with the CFP/YFP-type FRET biosensor gene for Rho GTPases, using the PiggyBac retrotransposon-mediated gene transfer system, and then cultured for $2 \mathrm{w}$ with $10 \mu \mathrm{g} \mathrm{ml}^{-1}$ blasticidin $\mathrm{S}$ to select for vSMCs stably expressing the FRET biosensor (Raichu-Rac1, RhoA or Cdc42/vSMC). Next, a KDR system for vSMCs expressing Raichu-Rac1, Raichu-RhoA or Rhaicu-Cdc42 was established as described above, except for the use of an adenovirus encoding nontagged Pdlim5 (WT, S177A and S177D)-T2A-mCherry instead of EGFP-tagged Pdlim5 (Raichu-Rac1, RhoA or Cdc42/KDR-Pdlim5-T2A-mCherry). Forty-eight hours after the transduction of Pdlim5-T2A-mCherry, Raichu-Rac1, RhoA or Cdc42/KDR-Pdlim5-T2A-mCherry cells were re-plated on collagen-coated glassbase dishes. All experiments were performed at $37^{\circ} \mathrm{C}$ in a $5 \% \mathrm{CO}_{2}$ atmosphere using a heating chamber. Dual images for CFP and YFP were obtained on a laser scanning microscope (LSM710, Zeiss) using an excitation wavelength of $405 \mathrm{~nm}$ and emission bandpass filters of $458-510 \mathrm{~nm}$ for CFP and $517-598 \mathrm{~nm}$ for YFP. After background subtraction, FRET/CFP ratio images were created using the LSM software ZEN2011 (Zeiss) and displayed as an intensity-modulated display image. For quantitative FRET analysis, the FRET/CFP ratio of each cell was calculated by dividing the fluorescence intensity of YFP by that of CFP over the total cellular area.

GST pull-down assay. For identification of proteins associated with Pdlim5, $20 \mu \mathrm{g}$ of purified GST-tagged Pdlim5 WT or S177D-Pdlim5 was immobilized on
glutathione-Sepharose beads and incubated at $4{ }^{\circ} \mathrm{C}$ for $2 \mathrm{~h}$ with cell lysate from $2 \times 10^{9}$ U937 cells in lysis buffer ( $30 \mathrm{mM}$ MOPS pH 7.5, $150 \mathrm{mM} \mathrm{NaCl}, 0.5 \%$ Triton-X100, $1.5 \mathrm{mM} \mathrm{MgCl}$, $1 \mathrm{mM}$ EGTA, $1 \mathrm{mM}$ DTT and protease inhibitor cocktail). After three washes of the beads with lysis buffer, associated proteins were eluted in elution buffer ( $30 \mathrm{mM}$ MOPS $\mathrm{pH} 7.5,500 \mathrm{mM} \mathrm{NaCl}, 0.5 \%$ Triton-X100 $1.5 \mathrm{mM} \mathrm{MgCl}_{2}, 1 \mathrm{mM}$ EGTA, $1 \mathrm{mM}$ DTT and protease inhibitor cocktail) and then subjected to silver staining and high-sensitivity shotgun liquid chromatographymass spectrometry (LTQ Orbitrap ELITE, Thermo Scientific).

F-actin binding assay of AMPK. F-actin binding assays were performed using the Actin Binding Protein Biochem Kit (BK001, Cytoskeleton, Inc.) according to the manufacturer's protocol, with minor modifications. Briefly, $1 \mu \mathrm{l}$ of F-actin $(21 \mu \mathrm{M}$ actin) in actin polymerization buffer $(100 \mathrm{mM} \mathrm{KCl}, 2 \mathrm{mM} \mathrm{MgCl}, 0.5 \mathrm{mM}$ ATP, $0.2 \mathrm{mM}$ Tris-HCl, $\mathrm{pH} 8.0)$ was mixed with $0.5 \mu \mathrm{l}$ of purified AMPK $\left(200 \mathrm{ng}^{-1} \mathrm{l}^{-1}\right)$ in the presence or absence of GST-tagged WT-Pdlim 5 and $1 \mu \mathrm{l}$ of $\alpha$-actinin $\left(1 \mu \mathrm{g} \mu \mathrm{l}^{-1}\right)$ in $60 \mu \mathrm{l}$ reaction volume, incubated for $1 \mathrm{~h}$ at $24^{\circ} \mathrm{C}$ and then centrifuged at $150,000 \mathrm{~g}$ for $1.5 \mathrm{~h}$ at $24^{\circ} \mathrm{C}$, to pellet the F-actin polymer and associated proteins. Equal amounts of pellet (P) and supernatant (S) were resolved by SDSPAGE and analysed by immunoblotting with the indicated antibodies.

Statistical analyses. Box plots show the entire population. Other data are expressed as means \pm s.e.m. Two-tailed Student's $t$-test was used to analyse differences between two groups. Differences among multiple groups were compared by one-way analysis of variance, followed by a post-hoc comparison with Dunnett's method using the JMP 8.0.1 software (SAS Institute Inc., Cary, NC, USA). $P<0.01$ was considered to indicate statistically significant differences.

\section{References}

1. Carling, D., Mayer, F. V., Sanders, M. J. \& Gamblin, S. J. AMP-activated protein kinase: nature's energy sensor. Nat. Chem. Biol. 7, 512-518 (2011).

2. Lee, J. H. et al. Energy-dependent regulation of cell structure by AMP-activated protein kinase. Nature 447, 1017-1020 (2007).

3. Zhang, L., Li, J., Young, L. H. \& Caplan, M. J. AMP-activated protein kinase regulates the assembly of epithelial tight junctions. Proc. Natl Acad. Sci. USA 103, 17272-17277 (2006)

4. Nakano, A. et al. AMPK controls the speed of microtubule polymerization and directional cell migration through CLIP-170 phosphorylation. Nat. Cell Biol. 12, 583-590 (2010).

5. Bae, H. B. et al. AMP-activated protein kinase enhances the phagocytic ability of macrophages and neutrophils. FASEB J. 25, 4358-4368 (2011).

6. Kondratowicz, A. S., Hunt, C. L., Davey, R. A., Cherry, S. \& Maury, W. J. AMPactivated protein kinase is required for the macropinocytic internalization of ebolavirus. J. Virol. 87, 746-755 (2013).

7. Parsons, J. T., Horwitz, A. R. \& Schwartz, M. A. Cell adhesion: integrating cytoskeletal dynamics and cellular tension. Nat. Rev. Mol. Cell Biol. 11, 633-643 (2010).

8. Wu, Y. I. et al. A genetically encoded photoactivatable Rac controls the motility of living cells. Nature 461, 104-108 (2009).

9. Sanz-Moreno, V. et al. Rac activation and inactivation control plasticity of tumor cell movement. Cell 135, 510-523 (2008).

10. Campellone, K. G. \& Welch, M. D. A nucleator arms race: cellular control of actin assembly. Nat. Rev. Mol. Cell Biol. 11, 237-251 (2010).

11. Ridley, A. J. Life at the leading edge. Cell 145, 1012-1022 (2011).

12. Dihu, J. B., Abudayyeh, I., Saudye, H. A. \& Gurujal, R. Cilostazol: a potential therapeutic option to prevent in-stent restenosis. J. Am. Coll. Cardiol. 57, 2035-2036 (2011).

13. Nissen, S. E. et al. Comparison of pioglitazone vs glimepiride on progression of coronary atherosclerosis in patients with type 2 diabetes: the PERISCOPE randomized controlled trial. JAMA 299, 1561-1573 (2008).

14. Bailey, C. J. Metformin: effects on micro and macrovascular complications in type 2 diabetes. Cardiovasc. Drugs Ther. 22, 215-224 (2008).

15. Esfahanian, N. et al. Effect of metformin on the proliferation, migration, and MMP-2 and -9 expression of human umbilical vein endothelial cells. Mol. Med. Rep. 5, 1068-1074 (2012).

16. Ridley, A. J. et al. Cell migration: integrating signals from front to back. Science 302, 1704-1709 (2003).

17. Gruzman, A., Babai, G. \& Sasson, S. Adenosine monophosphate-activated protein kinase (AMPK) as a new target for antidiabetic drugs: a review on metabolic, pharmacological and chemical considerations. Rev. Diabetic Studies 6, 13-36 (2009).

18. te Velthuis, A. J. \& Bagowski, C. P. PDZ and LIM domain-encoding genes: molecular interactions and their role in development. Sci. World J. 7, 14701492 (2007).

19. Zheng, Q. \& Zhao, Y. The diverse biofunctions of LIM domain proteins: determined by subcellular localization and protein-protein interaction. Biol. Cell 99, 489-502 (2007). 
20. Lasorella, A. \& Iavarone, A. The protein ENH is a cytoplasmic sequestration factor for Id 2 in normal and tumor cells from the nervous system. Proc. Natl Acad. Sci. USA 103, 4976-4981 (2006).

21. Yoshizaki, H. et al. Activity of Rho-family GTPases during cell division as visualized with FRET-based probes. J. Cell Biol. 162, 223-232 (2003).

22. Feng, Q., Baird, D. \& Cerione, R. A. Novel regulatory mechanisms for the Dbl family guanine nucleotide exchange factor Cool-2/alpha-Pix. EMBO J. 23, 3492-3504 (2004).

23. Matsuda, C. et al. Affixin activates Racl via betaPIX in C2C12 myoblast. FEBS Lett. 582, 1189-1196 (2008).

24. Viollet, B. et al. AMPK: Lessons from transgenic and knockout animals. Front. Biosci. (Landmark Ed) 14, 19-44 (2009).

25. Laderoute, K. R. et al. $5^{\prime}$-AMP-activated protein kinase (AMPK) is induced by low-oxygen and glucose deprivation conditions found in solid-tumor microenvironments. Mol. Cell Biol. 26, 5336-5347 (2006).

26. Faubert, B. et al. AMPK is a negative regulator of the Warburg effect and suppresses tumor growth in vivo. Cell Metab. 17, 113-124 (2013).

27. Cheng, H. et al. Loss of enigma homolog protein results in dilated cardiomyopathy. Circ. Res. 107, 348-356 (2010).

28. Horiuchi, Y. et al. Experimental evidence for the involvement of PDLIM5 in mood disorders in hetero knockout mice. PLOS ONE 8, e59320 (2013).

29. Rosenberger, G., Jantke, I., Gal, A. \& Kutsche, K. Interaction of alphaPIX (ARHGEF6) with beta-parvin (PARVB) suggests an involvement of alphaPIX in integrin-mediated signaling. Hum. Mol. Genet. 12, 155-167 (2003).

30. Mishima, W. et al. The first $\mathrm{CH}$ domain of affixin activates $\mathrm{Cdc} 42$ and $\mathrm{Racl}$ through alphaPIX, a Cdc42/Rac1-specific guanine nucleotide exchanging factor. Genes Cells 9, 193-204 (2004).

31. Cabodi, S., del Pilar Camacho-Leal, M., Di Stefano, P. \& Defilippi, P. Integrin signalling adaptors: not only figurants in the cancer story. Nat. Rev. Cancer 10, 858-870 (2010).

32. Wickstrom, S. A., Lange, A., Montanez, E. \& Fassler, R. The ILK/PINCH/parvin complex: the kinase is dead, long live the pseudokinase! EMBO J. 29, 281-291 (2010).

33. Yamaji, S. et al. Affixin interacts with alpha-actinin and mediates integrin signaling for reorganization of F-actin induced by initial cell-substrate interaction. J. Cell Biol. 165, 539-551 (2004).

34. Nakagawa, N. et al. ENH, containing PDZ and LIM domains, heart/skeletal muscle-specific protein, associates with cytoskeletal proteins through the PDZ domain. Biochem. Biophys. Res. Commun. 272, 505-512 (2000).

35. Michaelson, D. et al. Differential localization of Rho GTPases in live cells: regulation by hypervariable regions and RhoGDI binding. J. Cell Biol. 152, 111-126 (2001)

36. Johnston, S. A., Bramble, J. P., Yeung, C. L., Mendes, P. M. \& Machesky, L. M. Arp2/3 complex activity in filopodia of spreading cells. BMC Cell Biol. 9, 65 (2008).

37. Suraneni, P. et al. The Arp2/3 complex is required for lamellipodia extension and directional fibroblast cell migration. J. Cell Biol. 197, 239-251 (2012).

38. Wu, C. et al. Arp2/3 is critical for lamellipodia and response to extracellular matrix cues but is dispensable for chemotaxis. Cell 148, 973-987 (2012).

39. Hotulainen, P. \& Lappalainen, P. Stress fibers are generated by two distinct actin assembly mechanisms in motile cells. J. Cell Biol. 173, 383-394 (2006).

40. Sander, E. E., ten Klooster, J. P., van Delft, S., van der Kammen, R. A. \& Collard, J. G. Rac downregulates Rho activity: reciprocal balance between both
GTPases determines cellular morphology and migratory behavior. J. Cell Biol. 147, 1009-1022 (1999).

41. Chauhan, B. K., Lou, M., Zheng, Y. \& Lang, R. A. Balanced Racl and RhoA activities regulate cell shape and drive invagination morphogenesis in epithelia. Proc. Natl Acad. Sci. USA 108, 18289-18294 (2011).

42. Kovar, D. R., Harris, E. S., Mahaffy, R., Higgs, H. N. \& Pollard, T. D. Control of the assembly of ATP- and ADP-actin by formins and profilin. Cell 124, 423-435 (2006).

43. Aoki, K., Komatsu, N., Hirata, E., Kamioka, Y. \& Matsuda, M. Stable expression of FRET biosensors: a new light in cancer research. Cancer Sci. 103, 614-619 (2012).

\section{Acknowledgements}

This research was supported by the Japan Society for the Promotion of Science (JSPS) through the Funding Program for Next-Generation World-Leading Researchers (NEXT Program), which was initiated by the Council for Science and Technology Policy (CSTP) grants-in-aid from the Ministry of Health, Labour and Welfare of Japan; grants-in-aid from the Ministry of Education, Culture, Sports, Science and Technology of Japan; and grants-in-aid from the Japan Society for the Promotion of Science. This research was also supported by grants from the Japan Heart Foundation, the Japan Cardiovascular Research Foundation, the Japan Medical Association, the Japan Intractable Diseases Research Foundation, the Uehara Memorial Foundation, the Takeda Science Foundation, the Ichiro Kanehara Foundation, the Inoue Foundation for Science, the Mochida Memorial Foundation, a Heart Foundation/Novartis Grant for Research Award on Molecular and Cellular Cardiology, the Japan Foundation of Applied Enzymology, the Naito Foundation, the Banyu Foundation and Showa Houkoukai. We thank Hitoshi Miyagi, Kenichi Kondo (Olympus Corporation) and Masako Yamaguchi (Carl Zeiss Microscopy Corporation) for technical advice regarding microscopy; Saori Ikezawa, Eri Takata and Keiko Singu for technical assistance; and Yuko Okada for secretarial support.

\section{Authors contributions}

Y.Y. and O.T. designed and conducted the study, performed most of the experiments and wrote the manuscript. S.T. designed and wrote the manuscript. A.N. discussed the results and helped to write the manuscript. H.K. and K.T. performed the proteomic analysis. H.K. conducted and supported the biological experiments. N.I. performed the biochemical experiments and helped to generate plasmids. S.H., S.Y., Y.S., K.M. and Y.A. discussed the results and reviewed the manuscript. Y.L. H.A., M.A. and T.M. discussed the results. M.K. supervised all work.

\section{Additional information}

Supplementary Information accompanies this paper at http://www.nature.com/ naturecommunications

Competing financial interests: The authors declare no competing financial interests.

Reprints and permission information is available online at http://npg.nature.com/ reprintsandpermissions/

How to cite this article: Yan, Y. et al. Augmented AMPK activity inhibits cell migration by phosphorylating the novel substrate Pdlim5. Nat. Commun. 6:6137 doi: 10.1038/ ncomms7137 (2015).

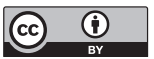

This work is licensed under a Creative Commons Attribution 4.0 International License. The images or other third party material in this article are included in the article's Creative Commons license, unless indicated otherwise in the credit line; if the material is not included under the Creative Commons license, users will need to obtain permission from the license holder to reproduce the material. To view a copy of this license, visit http://creativecommons.org/licenses/by/4.0/ 